\title{
Yangian-invariant spin models and Fibonacci numbers
}

\author{
Federico Finkel, Artemio González-López \\ Departamento de Física Teórica II, Universidad Complutense de Madrid, 28040 Madrid, Spain
}

\begin{abstract}
We study a wide class of finite-dimensional $\operatorname{su}(m \mid n)$-supersymmetric models closely related to the representations of the Yangian $Y(\mathrm{gl}(m \mid n))$ labeled by border strips. We quantitatively analyze the degree of degeneracy of these models arising from their Yangian invariance, measured by the average degeneracy of the spectrum. We compute in closed form the minimum average degeneracy of any such model, and show that in the non-supersymmetric case it can be expressed in terms of generalized Fibonacci numbers. Using several properties of these numbers, we show that (except in the simpler su(1|1) case) the minimum average degeneracy grows exponentially with the number of spins. We apply our results to several well-known spin chains of HaldaneShastry type, quantitatively showing that their degree of degeneracy is much higher than expected for a generic Yangian-invariant spin model. Finally, we show that the set of distinct levels of a Yangian-invariant spin model is described by an effective model of quasi-particles. We study this effective model, discussing its connections to one-dimensional anyons and properties of generalized Fibonacci numbers.
\end{abstract}

Keywords: Yangian symmetry, integrable spin chains, Fibonacci numbers, anyons

\section{Introduction}

In this paper we shall consider a general class of finite-dimensional quantum models which by construction are invariant under the Yangian algebra $Y(\operatorname{gl}(m \mid n))$. The key to the Yangian invariance of these models is their connection to certain finite-dimensional representations of $Y(\operatorname{gl}(m \mid n))$ labeled by a type of skew Young diagrams, the so called border strips [1, 2]. These representations play a fundamental role in the study of the integrable two-dimensional conformal field theory related to the latter models, namely the $\operatorname{su}(m \mid n)$ WZNW model at level 1 [3- 5]. The models we shall be interested in, which we shall refer to as Yangian-invariant $\operatorname{su}(m \mid n)$ spin models, are characterized by the fact that their Hilbert space is a direct sum of the irreducible representations of $Y(\mathrm{gl}(m \mid n))$ labeled by border strips with exactly $N$ boxes, where $N$ is the number of spins. The simplest examples of these models are certain integrable spin chains with long range interactions invariant under the Yangian for a finite number of spins, namely the $\operatorname{su}(m \mid n)$ supersymmetric versions of the Haldane-Shastry [6-8] and Polychronakos-Frahm [911] chains; see, e.g., [1, 3, 5, 12, 13].

Email addresses: ffinkel@ucm.es (Federico Finkel), artemio@ucm.es (Artemio González-López) 
As is well known, the Haldane-Shastry (HS) spin chain is a circular (translationally invariant) chain with equally spaced sites, the interactions between any two spins being inversely proportional to the square of their chord distance. This model appears in connection with a wide variety of topics in theoretical and mathematical physics, including one-dimensional anyons [3, 5, 8, 14], conformal field theory [15-18], quantum chaos [19-22], quantum information theory [23], and quantum integrability [12, 24, 25]. A distinctive feature of the HS chain is the fact that it can be obtained from a dynamical model, namely the spin Sutherland (trigonometric) model [26-28], in the strong coupling limit [9]. By applying the same procedure to the spin Calogero (rational) [29, 30] and Inozemtsev (hyperbolic) [31] models, one obtains the Polychronakos-Frahm (PF) and Frahm-Inozemtsev (FI) [32] spin chains, which share many properties with the original HS chain. In fact, although the Yangian symmetry of the FI chain has not been rigorously established, we shall see in Section 2 that it is isospectral to a Yangian-invariant spin model.

It is clear by its very definition that the class of Yangian-invariant spin models encompasses a wide range of quantum systems. For instance, apart from the integrable spin chains mentioned above, it includes the family of one-dimensional vertex models studied in Refs. [13, 33]. A common feature shared by all Yangian-invariant spin models is the high degeneracy of their spectrum, stemming from the Yangian symmetry. This statement, however, is far from precise, and does not shed any light on whether a certain model in this class has additional degeneracy due to its particular form. The main aim of this paper is precisely to perform a quantitative analysis of the degeneracy inherent to all Yangian-invariant spin models. To this end, we shall use as a concrete measure of the degree of degeneracy of a finite-dimensional quantum system its average degeneracy, defined as the quotient of the dimension of its Hilbert space by the number of distinct energy levels. We shall derive closed-form expressions for the average degeneracy of a "generic" Yangian-invariant su $(m \mid n)$ spin model, both in the supersymmetric $(m n \neq 0)$ and non-supersymmetric cases. In fact, these expressions provide a lower bound for the average degeneracy of any Yangian-invariant spin model, and thus can be used as a practical test for ruling out that a particular quantum system belongs to this class.

As it turns out, the behavior of this lower bound (which we have termed "minimum average degeneracy") is rather different in the supersymmetric and non-supersymmetric cases. In the former case, the minimum average degeneracy is given by a simple power law in terms of the dimension $m+n$ of the one-particle Hilbert space (cf. Eq. (17)). Far more interestingly, in the non-supersymmetric case the minimum average degeneracy is expressed in terms of generalized Fibonacci numbers [34], which reduce to the standard ones for spin $1 / 2$ ( $m$ or $n$ equal to 2). Using standard properties of the generalized Fibonacci numbers, we derive the asymptotic behavior as the number of spins tends to infinity of the minimum average degeneracy in the non-supersymmetric case. We find that the leading behavior is again a power law involving the largest root (in module) of the characteristic polynomial of the generalized Fibonacci numbers.

Another goal of this work is to ascertain to what degree the average degeneracy of the three spin chains of Haldane-Shastry type (i.e., the HS, PF and FI chains) behaves as expected for a generic Yangian-invariant spin model. Contrary to popular belief, we find out that this is actually not the case. More precisely, we show that the number of distinct levels of these chains grows polynomially with the number of spins, whereas for generic Yangian-invariant spin models this growth is exponential. As explained in detail in Section 5, the ultimate reason for this different behavior is the fact that spin chains of HS type possess a polynomial dispersion relation. As a matter of fact, our results apply as well to the whole family of vertex models studied in Refs. [13, 33], which also have this property. Moreover, when the coefficients of a polynomial dispersion relation are rational numbers (as is the case for the HS and PF chains, and for the FI 
chain when its parameter is rational) we show that the number of distinct levels behaves as $N^{k+1}$, where $k$ is the total degree of the dispersion relation.

Another aspect of Yangian-invariant spin models that we shall analyze in this paper is their connection with one-dimensional anyons. In fact, it is well known that spin chains of HS type provide one of the simplest realizations of anyons in one dimension via Haldane's fractional statistics [14]. For instance, as shown in the latter reference, the spectrum of the su(2) HS chain is the same as that of a system of spin $1 / 2$ anyons with exclusion parameter $g=1 / 2$. This result was essentially extended to the $\operatorname{su}(m)$ case in [5], with $g=1 / m$. In this paper we focus instead on the set of distinct energy levels of an $\mathrm{su}(m)$ Yangian-invariant spin model, and show that it can be obtained from the spectrum of an effective model of quasi-particles corresponding to the 1's in a Haldane motif [3]. In particular, in the su(2) case this effective model is simply a system of anyons with $g=2$. In the general $\operatorname{su}(m)$ case we compute in closed form the statistical weights of these effective models, and use them to derive in a novel way several combinatorial identities for generalized Fibonacci numbers.

This paper is organized as follows. In Section 2 we review the description of the representations of the Yangian $Y(\mathrm{gl}(m \mid n))$ labeled by border strips, and give a precise definition of Yangian-invariant $\operatorname{su}(m \mid n)$ spin models. We also recall the definitions of the $\operatorname{su}(m \mid n)$ spin chains of HS type, and discuss their spectra and dispersion relations. Section 3 is devoted to the computation of the minimum average degeneracy of an arbitrary Yangian-invariant spin model, in both the supersymmetric and non-supersymmetric cases. We apply our results to the $\operatorname{su}(m \mid n)$ supersymmetric version of Inozemtsev's elliptic chain [35], showing that in the su(2), su(3) and $\mathrm{su}(2 \mid 1)$ (or equivalently su(1|2)) cases it is not a Yangian invariant spin model for a wide range of values of $N$. Using several properties of the generalized Fibonacci numbers, we also determine the asymptotic behavior of the minimum average degeneracy of a Yangian-invariant spin model. In Section 4 we study the average degeneracy of Yangian-invariant spin models that are also translationally invariant, like the Haldane-Shastry chain. As an example, we consider the su(1|1) Inozemtsev chain, which is known to be (isospectral to) a Yangian- and translationally-invariant spin model [36]. As mentioned above, in Section 5 we present a detailed analysis of the average degeneracy of spin chains of HS type and, more generally, of Yangian-invariant spin models with a polynomial dispersion relation. In Section 6 we introduce the effective models describing the set of distinct levels of a non-supersymmetric Yangian-invariant spin model, and discuss their interpretation in terms of anyons and their connection with several identities satisfied by generalized Fibonacci numbers. The paper ends with a brief section in which we summarize our main results.

\section{Yangian-invariant spin models}

In this section we shall provide a precise definition of the class of Yangian-invariant models on which we shall focus in this paper. For the reader's convenience, we shall begin by recalling the precise definition of the Yangian $Y(\mathrm{gl}(m \mid n))$. Following the original paper [37] and Ref. [38], we first give an explicit matrix realization of the Lie superalgebra $\operatorname{gl}(m \mid n)$. To this end, let us introduce a $\mathbb{Z}_{2}$ grading $p$ in the vector space $\mathbb{C}^{m+n}$ by setting $p(v)=0$ if $v \in \mathbb{C}^{m} \times\{0\}$ and $p(v)=1$ if $v \in\{0\} \times \mathbb{C}^{n}$. This induces a natural grading in the space $M_{m+n}(\mathbb{C})$ of $(m+n) \times(m+n)$ complex matrices given by

$$
\operatorname{deg}\left(E^{\alpha \beta}\right)=p(\alpha)+p(\beta),
$$

where $p(\alpha) \equiv p\left(e_{\alpha}\right),\left\{e_{1}, \ldots, e_{m+n}\right\}$ being the canonical basis of $\mathbb{C}^{m+n}$, and $E^{\alpha \beta}$ denotes the matrix whose only nonzero entry is a 1 in the $\alpha$-th row and $\beta$-th column. By definition, the Lie 
superalgebra $\operatorname{gl}(m \mid n)$ is the vector space $M_{m+n}(\mathbb{C})$ with the the graded commutator defined by the customary formula

$$
[A, B]_{ \pm}=A B-(-1)^{\operatorname{deg} A+\operatorname{deg} B} B A
$$

on matrices with well-defined degree, and extended by linearity to the whole space. The Yangian $Y(\mathrm{gl}(m \mid n))$ is then defined by means of the solution of the Yang-Baxter equation given by the $R$-matrix

$$
R(u)=u-h P,
$$

where $u$ is the spectral parameter, $h \in \mathbb{C}$ and $P$ is the graded permutation operator defined by

$$
P\left(e_{\alpha} \otimes e_{\beta}\right)=(-1)^{p(\alpha) p(\beta)} e_{\beta} \otimes e_{\alpha} .
$$

More precisely, $Y(\mathrm{gl}(m \mid n))$ is the associative (complex) superalgebra with generators

$$
T_{\alpha \beta}^{(k)}, \quad \alpha, \beta=1, \ldots, m+n, \quad k=1,2, \ldots,
$$

defined as follows. Let

$$
T(u)=\sum_{\alpha, \beta=1}^{m+n} T_{\alpha \beta}(u) E^{\alpha \beta} \in M_{m+n}(Y(\operatorname{gl}(m \mid n))),
$$

where the matrix elements $T_{\alpha \beta}(u)$ are defined by

$$
T_{\alpha \beta}(u)=\delta_{\alpha \beta}+\sum_{k=1}^{\infty} T_{\alpha \beta}^{(k)} u^{-k} .
$$

We also introduce a $\mathbb{Z}_{2}$ grading in $Y(\operatorname{gl}(m \mid n))$ by setting

$$
\operatorname{deg}\left(T_{\alpha \beta}^{(k)}\right)=p(\alpha)+p(\beta),
$$

so that the matrix $T(u)$ and the identity matrix $\mathbb{I}=\sum_{\alpha=1}^{m+n} E^{\alpha \alpha}$ are both even. Given two even $(m+n) \times(m+n)$ matrices $A, B$ with matrix elements in $Y(\operatorname{gl}(m \mid n))$, we define their graded tensor product by

$$
(A \otimes B)_{i j, k l}=(-1)^{\left(p_{i}+p_{k}\right) p_{j}} A_{i k} B_{k l} .
$$

The defining relation for the generators $T_{\alpha \beta}^{(k)}$ are then given by the equation

$$
R(u-v)^{1} T(u){ }^{2} T(v)={ }^{2} T(v){ }^{1} T(u) R(u-v),
$$

where the matrix $R$ is defined by Eq. (1) and we have used the standard notation ${ }^{1} T(u)=T(u) \otimes \mathbb{I}$, ${ }^{2} T(u)=\mathbb{I} \otimes T(u)$. Using the above definition of graded tensor product, it is easy to check that the latter equation is equivalent to the following system for the currents $T_{\alpha \beta}(u)$ of the Yangian $Y(\operatorname{gl}(m \mid n))$ :

$$
\begin{aligned}
& (u-v)\left[T_{\alpha \beta}(u), T_{\gamma \delta}(v)\right]_{ \pm}=(-1)^{p_{\alpha} p_{\gamma}+p_{\beta} p_{\gamma}+p_{\alpha} p_{\beta}} h \cdot\left(T_{\gamma \beta}(u) T_{\alpha \delta}(v)-T_{\gamma \beta}(v) T_{\alpha \delta}(u)\right) \\
& \alpha, \beta, \gamma, \delta=1, \ldots, m+n .
\end{aligned}
$$

This system obviously determines the defining relations of the generators $T_{\alpha \beta}^{(k)}$ of the Yangian superalgebra $Y(\operatorname{gl}(m \mid n))$ through Eq. (2). In fact, it is well known that this algebra is actually generated by its lowest two generators $T_{\alpha \beta}^{(0)}$ and $T_{\alpha \beta}^{(1)}$. 
As mentioned in the Introduction, the models we shall deal with in this paper are associated to finite-dimensional representations of the Yangian $Y(\mathrm{gl}(\mathrm{m} \mid n))$ labeled by a particular class of skew Young diagrams, namely border strips, that we shall describe next. We shall start with a few preliminary definitions, following for the most part the notation in Ref. [1]. A skew Young diagram $\lambda / \mu$ is the set of boxes obtained by subtracting a Young diagram $\mu$ from a larger Young diagram $\lambda \supset \mu$. Such a diagram $\lambda / \mu$ is connected if for any pair of boxes $a, b \in \lambda / \mu$ there is a sequence of adjacent boxes starting with $a$ and ending with $b$. A border strip is a connected skew Young diagram not containing any $2 \times 2$ block of boxes. In other words, a border strip is a collection of boxes arranged in columns, such that the top box in each column is adjacent to the bottom box in the column to its right. Thus a border strip is determined by a vector $\mathbf{k} \equiv\left(k_{1}, \ldots, k_{r}\right)$, where $k_{i}$ is the number of boxes in the $i$-th column, counting from right to left; cf. Fig. 11 For this reason, from now on we shall usually identify a border strip with its corresponding vector of column lengths $\mathbf{k}$. An $\operatorname{su}(m \mid n)$ semi-standard tableau (SST) is a particular filling of the boxes in

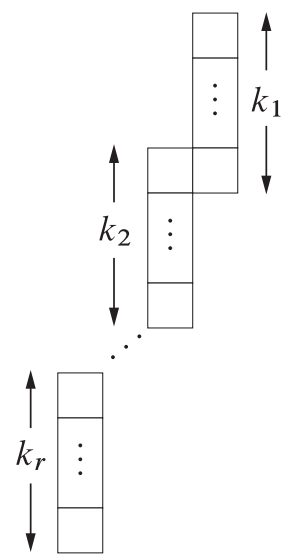

Figure 1: Border strip $\left(k_{1}, k_{2}, \ldots, k_{r}\right)$

a border strip $\mathbf{k}$ with integers in the range $-n,-n+1, \ldots, m-1$ according to the following two rules:

1. The integers in each column (read from top to bottom) and each row (read from left to right) form a nondecreasing sequence.

2. The nonnegative (respectively negative) integers in each row (resp. column) form an increasing sequence.

Remark 1. The use of the term $\operatorname{su}(m \mid n)$ in the previous definition is motivated by the fact that, as we shall explain in detail below, these tableaux appear in a natural way in the description of the spectrum of several well known spin chains possessing $\operatorname{su}(m \mid n)$ supersymmetry like, e.g., the Haldane-Shastry chain.

We can obviously identify an $\operatorname{su}(m \mid n)$ SST with the sequence

$$
\mathbf{s} \equiv\left(s_{1}, \ldots, s_{N}\right), \quad s_{i} \in\{-n,-n+1, \ldots, m-1\},
$$

of its integers read from top to bottom and from right to left; we shall call such a sequence the tableau's spin configuration. For instance, the tableau in Fig. 2 is an su(2|3) (or more generally 
$\operatorname{su}(m \mid n)$, with $m \geqslant 2$ and $n \geqslant 3)$ SST with spin configuration $\mathbf{s}=(-3,1,1,0,-2,-1,-1)$, associated to the border strip $\mathbf{k}=(3,1,2,1)$. We shall write $\mathbf{s} \in \mathbf{k}$ to indicate that $\mathbf{s}$ is the spin configuration of an $\operatorname{su}(m \mid n)$ SST whose underlying border strip is $\mathbf{k}$. We shall also say that two spin configurations $\mathbf{s}, \mathbf{s}^{\prime}$ are equivalent, and use the notation $\mathbf{s} \sim \mathbf{s}^{\prime}$, provided that $\mathbf{s}, \mathbf{s}^{\prime} \in \mathbf{k}$ for a certain border strip $\mathbf{k}$.

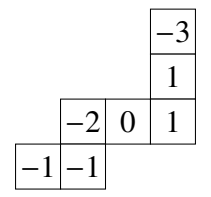

Figure 2: $\operatorname{su}(2 \mid 3)$ SST with spin configuration $\mathbf{s}=(-3,1,1,0,-2,-1,-1)$

As is well-known, the set of all border strips is in one-to-one correspondence with an important class of finite-dimensional irreducible representations of the Yangian $Y(\operatorname{gl}(m \mid n))$ whose carrier spaces (the so called tame modules) are irreducible under the action of a distinguished maximal abelian subalgebra of $Y(\operatorname{gl}(m \mid n))$ [1, 2]. Given a border strip $\mathbf{k}$, the dimension of the corresponding irreducible representation is the number of all possible $\operatorname{su}(m \mid n)$ SST associated with $\mathbf{k}$. It is clear that any vector $\mathbf{s} \in\{-n,-n+1, \ldots, m-1\}^{N}$ can be regarded as the spin configuration of the $\operatorname{su}(m \mid n)$ tableau constructed by placing the first component $s_{1}$ at the top of the first (rightmost) column, and then placing each component $s_{i}$ in the box below (respectively to the left) of the $(i-1)$-th box if $s_{i}>s_{i-1}$, or $s_{i}=s_{i-1} \geqslant 0$ (resp. $s_{i}<s_{i-1}$, or $\left.s_{i}=s_{i-1}<0\right)$. It immediately follows that the number of all $\operatorname{su}(m \mid n)$ SST with $N$ boxes coincides with the number $(m+n)^{N}$ of all spin configurations $\mathbf{s} \in\{-n,-n+1, \ldots, m-1\}^{N}$. This implies that

$$
\operatorname{dim} \bigoplus_{\mathbf{k} \in \mathcal{P}_{N}} V_{\mathbf{k}}(m \mid n)=(m+n)^{N},
$$

where $V_{\mathbf{k}}(m \mid n)$ denotes an irreducible $Y(\mathrm{gl}(m \mid n))$-module associated with the border strip $\mathbf{k}$ and $\mathcal{P}_{N}$ is the set of all partitions of the integer $N$ (with order taken into account). Thus the direct sum $\bigoplus_{\mathbf{k} \in \mathcal{P}_{N}} V_{\mathbf{k}}(m \mid n)$ has the same dimension as the Hilbert space of a spin chain with $N$ sites and two species of particles, one with $m$ and the other with $n$ internal degrees of freedom. In fact, there is an important class of Yangian-invariant models whose Hilbert space decomposes as the direct $\operatorname{sum} \bigoplus_{\mathbf{k} \in \mathcal{P}_{N}} V_{\mathbf{k}}(m \mid n)$, namely the su( $(m \mid n)$-supersymmetric Haldane-Shastry and PolychronakosFrahm chains [1, 4, 11, 12, 20, 39] that we shall now briefly describe.

To begin with, the Hilbert space of these chains is spanned by the canonical spin basis

$$
\left|s_{1}\right\rangle \otimes \cdots \otimes\left|s_{N}\right\rangle \equiv\left|s_{1}, \ldots, s_{N}\right\rangle \equiv|\mathbf{s}\rangle, \quad s_{i} \in\{-n,-n+1, \ldots, m-1\},
$$

where the $m$ bosonic (resp. $n$ fermionic) internal degrees of freedom of the $i$-th spin correspond to nonnegative (resp. negative) values of $s_{i}$. The action of the $\operatorname{su}(m \mid n)$-supersymmetric spin permutation operators $S_{i j}^{(m \mid n)}$ (with $i<j$ ) on the canonical spin basis is then defined by

$$
S_{i j}^{(m \mid n)}\left|s_{1}, \ldots, s_{i}, \ldots, s_{j}, \ldots, s_{N}\right\rangle=\sigma_{i j}(\mathbf{s})\left|s_{1}, \ldots, s_{j}, \ldots, s_{i}, \ldots, s_{N}\right\rangle,
$$

with $\sigma_{i j}(\mathbf{s})=-1$ when $s_{i}$ and $s_{j}$ are both fermionic, or when $s_{i}$ and $s_{j}$ are of opposite type and the number of fermionic spins occupying the sites $i+1, \ldots, j-1$ is odd. In terms of these operators, the Hamiltonians of the $\operatorname{su}(m \mid n)$ HS and PF chains can be taken as [20, 40]

$$
H=\sum_{1 \leqslant i<j \leqslant N} J_{i j}\left(1-S_{i j}^{(m \mid n)}\right),
$$


where

$$
J_{i j}= \begin{cases}\frac{1}{2} \sin ^{-2}(\pi(i-j) / N), & \text { for the HS chain, } \\ \left(\xi_{i}-\xi_{j}\right)^{-2}, & \text { for the PF chain, }\end{cases}
$$

and $\xi_{1}<\cdots<\xi_{N}$ are the zeros of the Hermite polynomial of degree $N$.

For the HS and PF chains, there is a simple explicit formula for the energy associated with the irreducible representation labeled by a border strip $\mathbf{k} \in \mathcal{P}_{N}$ that we shall now recall. To this end, it is convenient to introduce an alternative notation for the border strips in terms of the so-called motifs [3, 15]. More precisely, to a given a border strip $\mathbf{k} \in \mathcal{P}_{N}$ we shall associate the vector $\delta \in\{0,1\}^{N-1}$ with components

$$
\delta_{i}= \begin{cases}1, & i=k_{1}, k_{1}+k_{2}, \ldots, k_{1}+\cdots+k_{r-1}, \\ 0, & \text { otherwise }\end{cases}
$$

We shall call this vector $\delta$ the motif $f^{17}$ representing the border strip $\mathbf{k}$, and shall refer to the positions $K_{i} \equiv k_{1}+k_{2} \cdots+k_{i}$ of its nonzero components as its rapidities. For instance, for the border strip $\mathbf{k}=(3,1,2,1) \in \mathcal{P}_{7}$ in Fig. 2 the rapidities are 3, 4, 6, and thus the corresponding motif is $(0,0,1,1,0,1)$. For a given $\operatorname{su}(m \mid n)$ SST with spin configuration $\mathbf{s}$, we define $\delta(\mathbf{s})$ as the motif $\delta$ of the border strip determined by $\mathbf{s}$. It is immediate to convince oneself that

$$
\delta_{i}(\mathbf{s})= \begin{cases}1, & \text { if } s_{i+1}<s_{i} \text { or } s_{i}=s_{i+1}<0 \\ 0, & \text { otherwise }\end{cases}
$$

With these definitions, the spectrum of the $\operatorname{su}(m \mid n)$ HS and PF chains with $N$ sites (with the correct degeneracy for each level) can be shown to be the set of numbers [11, 13]

$$
\mathcal{E}_{N}(\mathbf{s})=\sum_{j=1}^{N-1} \varepsilon_{N}(j) \delta_{j}(\mathbf{s})
$$

with dispersion relation

$$
\varepsilon_{N}(j)= \begin{cases}j(N-j), & \text { for the HS chain } \\ j, & \text { for the PF chain. }\end{cases}
$$

In fact, a similar result holds for the (purely bosonic or fermionic) su( $m$ ) Frahm-Inozemtsev (FI) chain [32], whose Hamiltonian is of the form (3) with couplings

$$
J_{i j}=\frac{1}{2} \sinh ^{-2}\left(\zeta_{i}-\zeta_{j}\right) .
$$

Here $\mathrm{e}^{2 \zeta_{k}}$ denotes the $k$-th root of the generalized Laguerre polynomial $L_{N}^{\alpha-1}$, and $\alpha>0$ is a free parameter. In this case the spectrum is also given by Eq. [5], with dispersion relation [41]

$$
\varepsilon_{N}(j)=j(\alpha+j-1) .
$$

\footnotetext{
${ }^{1}$ In the original definition of Haldane, the motif is the sequence of the components of $\delta$ with a zero added at both ends.
} 
It can be shown that this formula for the spectrum holds as well in the $\operatorname{su}(m \mid n)$ case. In view of these facts, it is natural to conjecture that the $\operatorname{su}(m \mid n)$ FI chain is also invariant under the Yangian $Y(\operatorname{gl}(m \mid n))$, although to the best of our knowledge this result has not been rigorously proved.

We shall study in this paper the degeneracy of the spectrum of the class of $Y(\operatorname{gl}(m \mid n))$ invariant quantum systems whose Hilbert space $\mathcal{H}_{N}$ decomposes as the direct sum

$$
\mathcal{H}_{N}=\bigoplus_{\mathbf{k} \in \mathcal{P}_{N}} V_{\mathbf{k}}(m \mid n)
$$

of the irreducible representations of $Y(\operatorname{gl}(m \mid n))$ labeled by border strips with exactly $N$ boxes. We shall call any such system a Yangian-invariant $\operatorname{su}(m \mid n)$ spin model with $N$ sites. As we have just seen, this class includes the su( $m \mid n)$-supersymmetric HS and PF long-range spin chains. The Yangian invariance of these spin models and the decomposition (8) imply that their spectrum is of the form

$$
\mathcal{E}_{N}(\mathbf{s}), \quad \mathbf{s} \in\{-n,-n+1, \ldots, m-1\}^{N},
$$

where $\mathcal{E}_{N}$ is a real-valued function satisfying

$$
\mathbf{s} \sim \mathbf{s}^{\prime} \Longrightarrow \mathcal{E}_{N}(\mathbf{s})=\mathcal{E}_{N}\left(\mathbf{s}^{\prime}\right)
$$

Indeed, two spin configurations are equivalent if and only if they determine the same border strip $\mathbf{k}$, which labels a unique irreducible representation $V_{\mathbf{k}}(m \mid n)$ of the Yangian. Since obviously

$$
\mathbf{s} \sim \mathbf{s}^{\prime} \Longleftrightarrow \delta(\mathbf{s})=\delta\left(\mathbf{s}^{\prime}\right),
$$

we must have

$$
\mathcal{E}_{N}(\mathbf{s})=E_{N}(\delta(\mathbf{s}))
$$

Thus the spectrum of a Yangian-invariant spin model can be equivalently described by the function $E_{N}:\{0,1\}^{N-1} \rightarrow \mathbb{R}$ as the set of numbers

$$
E_{N}(\boldsymbol{\delta}(\mathbf{s})), \quad \mathbf{s} \in\{-n,-n+1, \ldots, m-1\}^{N} .
$$

We shall call $E_{N}$ the energy function of the system. It follows from Eq. (9) that the Hamiltonian of a Yangian-invariant spin model can be expressed as

$$
H=\sum_{\mathbf{k} \in \mathcal{P}_{N}} E_{N}(\boldsymbol{\delta}) \sum_{\mathbf{s} \in \mathbf{k}}|\mathbf{s}\rangle\langle\mathbf{s}|,
$$

where $\{|\mathbf{s}\rangle \mid \mathbf{s} \in \mathbf{k}\}$ is any orthonormal basis of the subspace $V_{\mathbf{k}}(m \mid n)$ and $\boldsymbol{\delta}$ denotes the motif corresponding to the border strip k. For instance, for the $\operatorname{su}(m \mid n)$ HS and PF chains the energy function is the linear functional

$$
E_{N}(\boldsymbol{\delta})=\sum_{j=1}^{N-1} \varepsilon_{N}(j) \delta_{j} .
$$

with $\varepsilon_{N}$ given by Eq. (6). In fact, more general Yangian-invariant spin models having a linear energy function 10 with polynomial dispersion relation $\varepsilon_{N}(j)$ have recently been studied in Refs. [13, 33].

We shall take Eq. (9) as the basis of our analysis of the spectrum of a Yangian-invariant spin model. For this reason, our results will also apply to models like the FI chain, whose Yangian invariance has not been established but which is known to possess a spectrum of the form (9) (with $E_{N}$ given by (7)-(10). 


\section{Average degeneracy and generalized Fibonacci numbers}

\subsection{Minimum average degeneracy}

The main goal of this section is to compute a lower bound for the average degeneracy of the spectrum 9] of a Yangian-invariant spin model with $N$ sites, defined by

$$
d_{N}=\frac{(m+n)^{N}}{\ell_{N}},
$$

where $\ell_{N}$ is the number of distinct energies. This is of course equivalent to finding an upper bound on $\ell_{N}$, a problem which we shall now address. The key observation in this respect is to note that, by Eq. (9), $\ell_{N}$ is obviously equal to the number of distinct values taken by the energy function $E_{N}(\delta)$, where $\delta$ ranges over the set

$$
\Delta_{N}(m \mid n)=\left\{\boldsymbol{\delta}(\mathbf{s}) \mid \mathbf{s} \in\{-n,-n+1, \ldots, m-1\}^{N}\right\} .
$$

of all valid $\operatorname{su}(m \mid n)$ motifs with $N-1$ components. Thus

$$
\ell_{N} \leqslant v_{N}(m \mid n),
$$

where $v_{N}(m \mid n)$ denotes the cardinal of the set $\Delta_{N}(m \mid n)$, and therefore

$$
d_{N} \geqslant d_{N, \min },
$$

where the minimum average degeneracy $d_{N, \min }$ is given by

$$
d_{N, \min }=\frac{(m+n)^{N}}{v_{N}(m \mid n)} \text {. }
$$

In fact, the minimum average degeneracy is strictly less than the average degeneracy if and only if the energy function $E_{N}$ is not injective; we shall say in this case that the Yangian-invariant spin model exhibits accidental degeneracy. We shall also say that a Yangian-invariant spin model is generic if it has no accidental degeneracy.

As we have just seen, in order to evaluate $d_{N, \min }$ in closed form we only need to compute the cardinal of the set (12). To this end, we shall next recall a simple characterization of all allowed $\operatorname{su}(m \mid n)$ motifs which shall be of fundamental importance in what follows. Consider, to begin with, the genuinely supersymmetric case $m n \neq 0$. It is easy to realize that in this case any sequence $\delta \in\{0,1\}^{N-1}$ is a valid motif, so that

$$
v_{N}(m \mid n)=2^{N-1}, \quad m n \neq 0 .
$$

Indeed, if $\delta \in\{0,1\}^{N-1}$ let $K_{1}, \ldots, K_{r-1}$ be the positions of the nonzero components of $\delta$, and define $k_{i}=K_{i}-K_{i-1}\left(\right.$ with $K_{0} \equiv 0$ and $\left.K_{r} \equiv N\right)$. Then the vector

$$
\mathbf{s}=(\underbrace{0, \ldots, 0}_{k_{1}}, \underbrace{-1,0, \ldots, 0}_{k_{2}}, \ldots, \underbrace{-1,0, \ldots, 0}_{k_{r}})
$$

is the spin configuration of an $\operatorname{su}(m \mid n)$ SST whose corresponding motif is $\delta$. Thus in this case we just have

$$
d_{N, \text { min }}=2\left(\frac{m+n}{2}\right)_{9}^{N}, \quad m n \neq 0 .
$$


Consider next the purely bosonic $\operatorname{su}(m \mid 0)$ case. It is clear that an $\operatorname{su}(m \mid 0)$ motif cannot contain a sequence of $m$ or more consecutive 1's, since the spin configuration of any tableau associated to such a motif would contain a sequence of $m+1$ or more distinct integers in the range $0,1, \ldots, m-1$. Conversely, suppose that the vector $\delta \in\{0,1\}^{N-1}$ contains no sequence of $m$ or more consecutive 1's. Such a $\delta$ can be written as a succession of sequences of consecutive 0 's and 1's of the form

$$
\boldsymbol{\delta}=(\underbrace{0, \ldots, 0}_{l_{1}}, \underbrace{1, \ldots, 1}_{n_{1}}, 0, \ldots)
$$

with $n_{i} \leqslant m-1$. It is immediate to check that the spin configuration

$$
\mathbf{s}=(\underbrace{m-1, \ldots, m-1}_{l_{1}+1}, m-2, \ldots, m-n_{1}, m-n_{1}-1, m-1, \ldots),
$$

where each entry is in the range $0,1, \ldots, m-1$ on account of the condition $n_{i} \leqslant m-1$, determines an $\operatorname{su}(m)$ SST whose corresponding motif is $\delta$. This shows that $\Delta_{N}(m \mid 0)$ is the set of all vectors $\delta \in\{0,1\}^{N-1}$ containing no sequences of $m$ or more consecutive 1 's. In exactly the same way it is proved that in the purely fermionic case $\Delta_{N}(0 \mid n)$ is the set of all vectors $\delta \in\{0,1\}^{N-1}$ containing no sequences of $n$ or more consecutive 0 's. It immediately follows from these two facts that the sets $\Delta_{N}(m \mid 0)$ and $\Delta_{N}(0 \mid m)$ are mapped bijectively into each other by the duality transformation

$$
\boldsymbol{\delta}=\left(\delta_{1}, \ldots, \delta_{N-1}\right) \in \Delta_{N}(m \mid 0) \mapsto \boldsymbol{\delta}^{\prime}=\left(1-\delta_{1}, \ldots, 1-\delta_{N-1}\right) \in \Delta_{N}(0 \mid m) .
$$

Hence

$$
v_{N}(m \mid 0)=v_{N}(0 \mid m),
$$

so that from now on we shall restrict ourselves without loss of generality to the purely bosonic case.

Remark 2. The duality transformation (18) actually arises from a mapping defined on spin configurations [38], valid also in the general supersymmetric case. More precisely, given an $\operatorname{su}(m \mid n)$ spin configuration $\mathbf{s}$ define the vector $\mathbf{s}^{\prime} \equiv\left(s_{1}^{\prime}, \ldots, s_{N}^{\prime}\right)$ by

$$
s_{i}^{\prime}=-s_{i}-1, \quad i=1, \ldots, N .
$$

It is immediate to check that $\mathbf{s}^{\prime}$ is a valid $\operatorname{su}(n \mid m)$ spin configuration, and that

$$
\delta\left(\mathbf{s}^{\prime}\right)=\delta(\mathbf{s})^{\prime},
$$

where $\delta^{\prime}$ is defined by Eq. (18). Consider two $\operatorname{su}(m \mid n)$ and $\operatorname{su}(n \mid m)$ spin models with the same energy function $E_{N}$. It is straightforward to show that the spectra of both models are mapped in a one-to-one way by the duality transformation

$$
E_{N}(\boldsymbol{\delta}) \mapsto E_{N}\left(\boldsymbol{\delta}^{\prime}\right)
$$

Indeed, it is obvious from Eq. 97 for the spectrum that the energies of both models are in a one-to-one correspondence under the transformation (21). Their respective degeneracies also coincide, since for a given $\operatorname{su}(m \mid n)$ motif $\delta$ we have

$$
\{\mathbf{s} \in\{-n,-n+1, \ldots, m-1\} \mid \boldsymbol{\delta}(\mathbf{s})=\boldsymbol{\delta}\}=\left\{\mathbf{s}^{\prime} \in\{-m,-m+1, \ldots, n-1\} \mid \boldsymbol{\delta}\left(\mathbf{s}^{\prime}\right)=\boldsymbol{\delta}^{\prime}\right\},
$$


on account of Eq. 20]. In particular, for the linear energy function (10) the relation between the corresponding spectra of two $\operatorname{su}(m \mid n)$ and $\operatorname{su}(n \mid m)$ models is simply given by

$$
E_{N}^{(n \mid m)}\left(\boldsymbol{\delta}^{\prime}\right)=E_{0}-E_{N}^{(m \mid n)}(\boldsymbol{\delta}),
$$

where

$$
E_{0}=\sum_{j=1}^{N-1} \varepsilon_{N}(j)
$$

is the maximum energy of the model(s) with a nonvanishing number of fermionic degrees of freedom.

In view of the above discussion, we need only compute the cardinal $v_{N}(m) \equiv v_{N}(m \mid 0)$ of the set of $\operatorname{su}(m) \equiv \operatorname{su}(m \mid 0)$ (purely bosonic) motifs. The key observation in this respect is that an $\operatorname{su}(m)$ motif $\boldsymbol{\delta}$ of length $N-1 \geqslant m$ must necessarily be of the form

$$
(\boldsymbol{\delta}^{(k)}, 0, \underbrace{1, \ldots, 1}_{k-1}), \quad k=1, \ldots, m,
$$

where $\delta^{(k)}$ is a valid $\operatorname{su}(m)$ motif of length $N-k-1$. This observation immediately leads to the recursion relation

$$
v_{N}(m)=\sum_{k=1}^{m} v_{N-k}(m), \quad N \geqslant m+1 .
$$

On the other hand, for $\operatorname{su}(m)$ motifs of length $N-1<m$ the restriction on the number of consecutive 1's is vacuous, so that

$$
\Delta_{N}(m \mid 0)=\{0,1\}^{N-1}, \quad N=1, \ldots, m,
$$

and therefore

$$
v_{N}(m)=2^{N-1}, \quad N=1, \ldots, m .
$$

The recursion relation 24a , together with the initial conditions 24b , uniquely determines $v_{N}(m)$ for arbitrary $N$. We shall next show that $v_{N}(m)$ can in fact be expressed in terms of the so-called $m$-generalized Fibonacci (in short, $m$-nacci) numbers $F_{n}^{(m)}$, defined [34] as the unique solution of the recursion relation

$$
F_{n}^{(m)}=\sum_{k=1}^{m} F_{n-k}^{(m)}, \quad n \geqslant m,
$$

with the initial conditions

$$
F_{0}^{(m)}=\cdots=F_{m-2}^{(m)}=0, \quad F_{m-1}^{(m)}=1 .
$$

Note, in particular, that for $m=2$ Eqs. 25 yield the standard Fibonacci numbers. Comparing Eqs. 24] with 25] it is straightforward to prove that

$$
v_{N}(m)=F_{N+m-1}^{(m)} .
$$


Indeed, it is obvious that the shifted $m$-nacci sequence $F_{N+m-1}^{(m)}$ satisfies the recursion relation 24a). Hence it suffices to show that (26) holds for $N=1, \ldots, m$. This is obviously true for $N=1$. Assuming that (26) also holds for $N=1, \ldots, k \leqslant m-1$, by Eqs. 25] we have

$$
F_{m+k}^{(m)}=F_{k}^{(m)}+\cdots+F_{m-1}^{(m)}+\sum_{j=0}^{k-1} F_{m+j}^{(m)}=1+\sum_{j=0}^{k-1} v_{j+1}(m)=1+\sum_{j=0}^{k-1} 2^{j}=2^{k}=v_{k+1}(m),
$$

so that (26) also holds for $N=k+1$. By induction, the latter equation is true for $N=1, \ldots, m$, thus establishing our claim. From Eq. (15) with $n=0$ we conclude that the minimum average degeneracy of a Yangian-invariant bosonic $\mathrm{su}(m)$ spin model is given by

$$
d_{N, \min } \equiv \frac{m^{N}}{F_{N+m-1}^{(m)}}
$$

By Eq. [19], the same is true for a purely fermionic su( $m)$ spin model.

The inequality (14) provides a very simple necessary condition for a quantum system to be a Yangian-invariant spin model, as we shall see in the following example.

Example 1. Consider the $\operatorname{su}(m \mid n)$ supersymmetric version of the Inozemtsev elliptic chain [35], with Hamiltonian

$$
H^{(m \mid n)}=\frac{1}{2} \sum_{1 \leqslant i<j \leqslant N} \frac{1-S_{i j}^{(m \mid n)}}{\operatorname{sn}^{2}\left(2(i-j) \frac{K}{N}\right)} .
$$

In the latter equation sn denotes Jacobi's elliptic sine with real period $4 K$, where $K \equiv K(k)$ is the complete elliptic integral of the first kind

$$
K(k)=\int_{0}^{\pi / 2} \frac{\mathrm{d} x}{\sqrt{1-k^{2} \sin ^{2} x}}
$$

and $k \in[0,1)$ is the elliptic modulus. As is well-known, for $k=0$ the latter model reduces to the $\operatorname{su}(m \mid n)$ HS chain (3)-(4) which, as we have seen in the previous section, is a Yangianinvariant spin model. On the other hand, for $k \rightarrow 1$ the Hamiltonian (28) is related to the $\operatorname{su}(m \mid n)$ Heisenberg chain, which does not possess Yangian invariance for a finite-number of sites. For $k \in(0,1)$, it is generally believed that the Hamiltonian 28$)$ is not invariant under $Y(\mathrm{gl}(m \mid n))$ for any finite value of $N$. We have numerically computed the average degeneracy of the spectrum of the model (28) with su(2), su(3) and su(2|1) $\operatorname{spin}^{2}$ (for $6 \leqslant N \leqslant 18$ in the first case and $6 \leqslant N \leqslant 12$ in the remaining ones). It turns our that for $k \in(0,1)$ the average degeneracy is practically independent of $k$, so that we shall restrict ourselves to the case $k^{2}=1 / 2$. As can be seen from Fig. 3. for $N \geqslant 8$ the average degeneracy of the model $(28)$ in the cases studied is clearly lower than the minimum average degeneracy of a Yangian-invariant spin model (cf. Eqs. (17) or 27). This shows that in these cases the chain 28) is not a Yangian-invariant spin model (see also Ref. [42] for the su(2) case).

\footnotetext{
${ }^{2}$ As shown in Ref. [38], the operators $S_{i j}^{(m \mid n)}$ and $-S_{i j}^{(n \mid m)}$ are unitarily equivalent, so that the spectra of $H^{(m \mid n)}$ and $-H^{(n \mid m)}$ differ by an additive constant. In particular, the average degeneracies of the $\operatorname{su}(m \mid n)$ and $\operatorname{su}(n \mid m)$ models 28 coincide.
} 

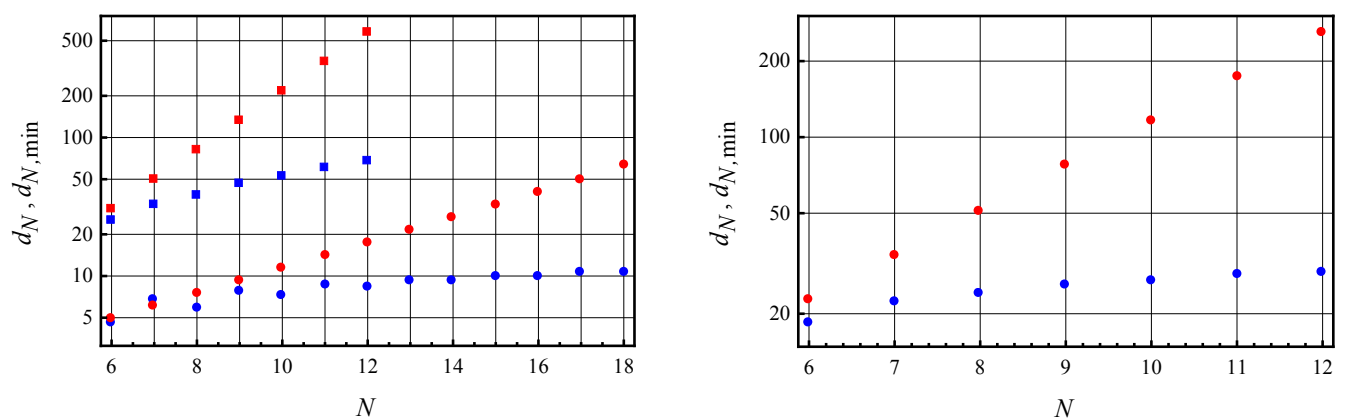

Figure 3: Left: logarithmic plot of the average degeneracy of the su(m) elliptic chain with $k^{2}=1 / 2$ and $m=2$ (blue circles) and $m=3$ (blue squares), compared to the minimum average degeneracy of an su( $m$ ) Yangian-invariant spin model with $m=2$ (red circles) and $m=3$ (red squares). Right: analogous plot for the su(2|1) elliptic chain with $k^{2}=1 / 2$ (blue circles) and the su(2|1) minimum average degeneracy (red circles).

\subsection{Asymptotic behavior of the minimum average degeneracy}

We shall next investigate the asymptotic behavior as $N$ tends to infinity of the minimum average degeneracy (27) of an $\mathrm{su}(m)$ Yangian-invariant spin model. To this end, note that 25a is a linear recursion relation with constant coefficients and characteristic polynomial

$$
p_{m}(\lambda)=\lambda^{m}-\sum_{k=1}^{m} \lambda^{m-k}
$$

It is shown in Ref. [34] that all the roots $\lambda_{i}^{(m)}(i=1, \ldots, m)$ of $p_{m}(t)$ are simple, and that moreover they can be labeled in such a way that

$$
\left|\lambda_{i}^{(m)}\right|<1, \quad i=1, \ldots, m-1 ; \quad 1<\lambda_{m}^{(m)} \equiv \lambda_{m}<2 .
$$

From the simple character of the roots of the characteristic polynomial it follows that the general solution of the recursion relation $(25 \mathrm{a})$ is of the form

$$
\sum_{i=1}^{m} c_{i}^{(m)}\left(\lambda_{i}^{(m)}\right)^{n}, \quad n=0,1, \ldots,
$$

where the coefficients $c_{i}^{(m)}$ are complex constants (independent of $N$ ). In order to obtain the $m$ nacci numbers, the coefficients $c_{i}^{(m)}$ must be chosen in such a way that 32 satisfies the initial conditions (25b), i.e.,

$$
\sum_{i=1}^{m} c_{i}^{(m)}\left(\lambda_{i}^{(m)}\right)^{n}=\delta_{n, m-1}, \quad n=0, \ldots, m-1 .
$$

Note that the coefficient matrix of this linear system is the Vandermonde matrix determined by the $m$ distinct roots of the characteristic polynomial $p_{m}(\lambda)$, and therefore the system has always a unique solution. In fact, it is straightforward to show that

$$
c_{i}^{(m)}=\prod_{j ; j \neq i}\left(\lambda_{i}^{(m)}-\lambda_{j}^{(m)}\right)^{-1},
$$


and therefore

$$
F_{n}^{(m)}=\sum_{i=1}^{m}\left(\lambda_{i}^{(m)}\right)^{n} \prod_{j ; j \neq i}\left(\lambda_{i}^{(m)}-\lambda_{j}^{(m)}\right)^{-1} .
$$

From this equation and Eq. 31] it follows that as $n \rightarrow \infty$ we have

$$
F_{n}^{(m)}=c_{m} \lambda_{m}^{n}+\mathrm{O}\left(\mathrm{e}^{-\kappa_{m} n}\right),
$$

with

$$
c_{m} \equiv c_{m}^{(m)}=\prod_{i=1}^{m-1}\left(\lambda_{m}-\lambda_{i}^{(m)}\right)^{-1}
$$

and

$$
\kappa_{m}=-\min _{1 \leqslant i \leqslant m-1} \log \left|\lambda_{i}^{(m)}\right|>0 .
$$

Thus as $N \rightarrow \infty$ the minimum average degeneracy behaves as

$$
d_{N, \min }=\gamma_{m}\left(\frac{m}{\lambda_{m}}\right)^{N}\left(1+\mathrm{O}\left(\mathrm{e}^{-\left(\kappa_{m}+\log \lambda_{m}\right) N}\right)\right),
$$

where the coefficient $\gamma_{m}$ (independent of $N$ ) is given by

$$
\gamma_{m}=\frac{1}{c_{m} \lambda_{m}^{m-1}}=\prod_{i=1}^{m-1}\left(1-\frac{\lambda_{i}^{(m)}}{\lambda_{m}}\right) .
$$

Since $\lambda_{m}<2$ (cf. Eq. (31)), it follows from Eq. (36) that $d_{N, \text { min }}$ grows exponentially as $N \rightarrow \infty$.

The coefficient $c_{m}$, and hence $\gamma_{m}$, can be expressed in terms of the root $\lambda_{m}$. Indeed, from Eq. (35) we have

$$
\frac{1}{c_{m}}=\prod_{i=1}^{m-1}\left(\lambda_{m}-\lambda_{i}^{(m)}\right)=\lim _{\lambda \rightarrow \lambda_{m}} \frac{p_{m}(\lambda)}{\lambda-\lambda_{m}}=p_{m}^{\prime}\left(\lambda_{m}\right) .
$$

In particular, from the previous expression it is obvious that $c_{m}$ is real and positive, as it should be on account of Eq. [34). Another useful expression for the coefficient $c_{m}$ is obtained by noting that, by Eq. 30,

$$
q_{m}(\lambda) \equiv(\lambda-1) p_{m}(\lambda)=\lambda^{m+1}-2 \lambda^{m}+1=(\lambda-1)\left(\lambda-\lambda_{m}\right) \prod_{i=1}^{m-1}\left(\lambda-\lambda_{i}^{(m)}\right),
$$

and therefore

$$
\frac{1}{c_{m}}=\frac{1}{\lambda_{m}-1} \lim _{\lambda \rightarrow \lambda_{m}} \frac{q_{m}(\lambda)}{\lambda-\lambda_{m}}=\frac{q_{m}^{\prime}\left(\lambda_{m}\right)}{\lambda_{m}-1}=\frac{(m+1) \lambda_{m}^{m}-2 m \lambda_{m}^{m-1}}{\lambda_{m}-1} .
$$

We thus arrive at the following remarkably simple expression for the coefficient $\gamma_{m}$ in Eq. (36):

$$
\gamma_{m}=\frac{\lambda_{m}^{1-m}}{c_{m}}=\frac{(m+1) \lambda_{m}-2 m}{\lambda_{m}-1}=m+1-\frac{m-1}{\lambda_{m}-1} .
$$

In particular, from Eqs. (31) and (38) we obtain the following upper and lower bounds on $\lambda_{m}$ :

$$
\frac{2 m}{m+1}<\lambda_{m}<2,
$$


which imply that

$$
\lim _{m \rightarrow \infty} \lambda_{m}=2 .
$$

In fact, it can be easily shown that the sequence $\left\{\lambda_{m}\right\}_{m=2}^{\infty}$ is monotonically increasing. Indeed,

$$
p_{m+1}\left(\lambda_{m}\right)=p_{m+1}\left(\lambda_{m}\right)-p_{m}\left(\lambda_{m}\right)=\lambda_{m}^{m}\left(\lambda_{m}-2\right)<0,
$$

so that $\lambda_{m}<\lambda_{m+1}$ since $\lambda_{m+1}$ is the largest root of the monic polynomial $p_{m+1}$.

On the other hand, since $\lambda=1$ is not a root of $p_{m}$, the characteristic equation $p_{m}(\lambda)=0$ can be written as

$$
\lambda^{m}-\frac{\lambda^{m}-1}{\lambda-1}=0
$$

or equivalently

$$
\lambda=2-\frac{1}{\lambda^{m}} .
$$

Hence

$$
\lim _{m \rightarrow \infty} m\left(2-\lambda_{m}\right)=\lim _{m \rightarrow \infty} \frac{m}{\lambda_{m}^{m}}=0
$$

and Eq. 38, implies that

$$
\gamma_{m}=\frac{\lambda_{m}-m\left(2-\lambda_{m}\right)}{\lambda_{m}-1} \underset{m \rightarrow \infty}{\longrightarrow} 2
$$

Thus, for $m \gg 1$ we have

$$
d_{N, \min } \simeq 2\left(\frac{m}{2}\right)^{N}, \quad m \gg 1,
$$

where the RHS is the minimum average degeneracy of an $\operatorname{su}(p \mid q)$ model with $p+q=m$ (cf. Eq. (17p).

It can be numerically checked that $\lambda_{m}$ and (to a lesser extent) $\gamma_{m}$ rapidly converge to 2 as $m$ tends to $\infty$ (see, e.g., Table 1 below). To confirm this observation analytically, it suffices to note that from Eq. 39] we have

$$
\frac{1}{\lambda_{m}^{m}}<\frac{1}{2^{m}}\left(1+\frac{1}{m}\right)^{m}<\frac{\mathrm{e}}{2^{m}}
$$

and therefore, by Eq. 41],

$$
2-\frac{\mathrm{e}}{2^{m}}<\lambda_{m}<2
$$

The upper bound on $\lambda_{m}$ implies that $\gamma_{m}<2$, while from the lower bound we easily obtain

$$
\gamma_{m}>m+1-\frac{m-1}{1-2^{-m} \mathrm{e}}=2-\frac{(m-1) \mathrm{e}}{2^{m}-\mathrm{e}}>2-\frac{m \mathrm{e}}{2^{m}} \quad \text { for } \quad m \geqslant 4 .
$$

Remark 3. Equation (37) for the coefficient $c_{m}$ can in fact be extended with exactly the same proof to the remaining coefficients $c_{i}^{(m)}(i=1, \ldots, m-1)$, i.e.,

$$
c_{i}^{(m)}=\frac{\lambda_{i}^{(m)}-1}{\left(\lambda_{i}^{(m)}\right)^{m-1}\left((m+1) \lambda_{i}^{(m)}-2 m\right)} .
$$


Inserting the latter equation into Eq. (32) provides an alternative simple derivation of Binet's formula for the $m$-nacci numbers [43, 44]

$$
F_{n}^{(m)}=\sum_{i=1}^{m} \frac{\lambda_{i}^{(m)}-1}{(m+1) \lambda_{i}^{(m)}-2 m}\left(\lambda_{i}^{(m)}\right)^{n-m+1} .
$$

Example 2. For $m=2$ the roots of the characteristic polynomial $p_{2}(\lambda)$ are

$$
\lambda_{1}=\frac{1}{2}(1-\sqrt{5}), \quad \lambda_{2}=\frac{1}{2}(1+\sqrt{5}),
$$

so that Eq. (33) reduces to the famous Binet formula for the Fibonacci numbers

$$
F_{n}^{(2)} \equiv F_{n}=\frac{1}{2^{n} \sqrt{5}}\left[(1+\sqrt{5})^{n}-(1-\sqrt{5})^{n}\right] .
$$

Thus the minimum average degeneracy (27) of a Yangian-invariant su(2) spin model is given by

$$
d_{N, \text { min }}=\frac{2^{2 N+1} \sqrt{5}}{(1+\sqrt{5})^{N+1}-(1-\sqrt{5})^{N+1}} \simeq \frac{\sqrt{5}}{2}(\sqrt{5}-1)^{N+1},
$$

in agreement with Eqs. (36) and 38.

In the case $m=3$, the characteristic polynomial has two complex conjugate roots of modulus less than one and a real root

$$
\lambda_{3}=\frac{1}{3}(1+\sqrt[3]{19+3 \sqrt{33}}+\sqrt[3]{19-3 \sqrt{33}}) \equiv \frac{1}{3}(1+r) \simeq 1.83929 .
$$

Using Eq. (38) we easily obtain

$$
\gamma_{3}=\frac{2(2 r-7)}{r-2} \simeq 1.61702
$$

so that for large $N$ the su(3) minimum average degeneracy behaves as

$$
d_{N, \min } \simeq 1.61702 \cdot 1.63107^{N}, \quad N \gg 1 .
$$

The exact formulas for $\lambda_{4}$ and $\gamma_{4}$ are too unwieldy to display, and for $m \geqslant 5$ the characteristic equation defining $\lambda_{m}$ cannot be solved in radicals. However, the numerical evaluation of $\lambda_{m}$ and $\gamma_{m}$ is straightforward; see Table 1 for a list of their values in the range $m=2, \ldots, 10$.

\section{Average degeneracy of translationally invariant models}

Several important examples of Yangian-invariant spin models possess the additional property of being translationally invariant. This is notably the case for the $\operatorname{su}(m \mid n)$ Haldane-Shastry spin chain (3)-(4), whose energy function is of the form (10) with $\varepsilon_{N}(j)=j(N-j)$. The dispersion relation of this chain obviously satisfies the identity

$$
\varepsilon_{N}(j)=\varepsilon_{N}(N-j),
$$




\begin{tabular}{|r|c|c|}
\hline$m$ & $\lambda_{m}$ & $\gamma_{m}$ \\
\hline 2 & 1.61803 & 1.38197 \\
3 & 1.83929 & 1.61702 \\
4 & 1.92756 & 1.76571 \\
5 & 1.96595 & 1.85899 \\
6 & 1.98358 & 1.91654 \\
7 & 1.99196 & 1.95139 \\
8 & 1.99603 & 1.97211 \\
9 & 1.99803 & 1.98420 \\
10 & 1.99902 & 1.99116 \\
\hline
\end{tabular}

Table 1: Values of $\lambda_{m}$ and $\gamma_{m}$ in the asymptotic formula 36 for $m=2, \ldots, 10$.

characteristic of translationally invariant models (on a lattice with unit spacing between consecutive sites) with a linear energy function. Indeed, for these models $\varepsilon_{N}(j)$ is naturally interpreted as the energy of a quasi-particle with momentum $2 \pi j / N$. Since the momentum is defined up to multiples of $2 \pi$ due to the translation invariance, the identity (43) simply expresses the fact that the energy does not depend on the sign of the momentum. When the energy function $E_{N}(\delta)$ is linear (cf. Eq. (10) and its dispersion relation satisfies Eq. (43), the spectrum can be expressed as

$$
E_{N}(\boldsymbol{\delta})=\sum_{j=1}^{\lfloor(N-1) / 2\rfloor} \varepsilon_{N}(j)\left(\delta_{j}+\delta_{N-j}\right)+(1-\pi(N)) \varepsilon_{N}(N / 2) \delta_{N / 2}, \quad \delta \in \Delta_{N}(m \mid n),
$$

where $\pi(N)$ is the parity of $N$ and $\lfloor\cdot\rfloor$ denotes the integer part. Thus in this case $E_{N}$ depends on $\delta$ only through the combination $\delta+\delta^{\mathrm{R}}$, where $\delta^{\mathrm{R}}$ is the reversed motif with components $\delta_{j}^{\mathrm{R}}=\delta_{N-j}$. Conversely, if the energy function is linear and verifies

$$
E_{N}(\boldsymbol{\delta})=f_{N}\left(\boldsymbol{\delta}+\boldsymbol{\delta}^{\mathrm{R}}\right)
$$

for some function $f_{N}$, then the corresponding dispersion relation $\varepsilon_{N}$ satisfies (43). Indeed, it suffices to impose (44) for motifs of the form $(0, \ldots, 0,1,0, \ldots, 0)$, which are allowed when $m>0$, or of the form $(1, \ldots, 1,0,1, \ldots, 1)$ when $n>0$. Motivated by these observations, we shall say that a general Yangian-invariant spin model is translationally invariant whenever its energy function (not necessarily linear) satisfies the identity (44). The purpose of this section is to study the minimum average degeneracy of such models.

By Eq. (44), the minimum average degeneracy of a translationally (and Yangian) invariant spin model is given by

$$
d_{N, \min }^{(\mathrm{s})}=\frac{(m+n)^{N}}{v_{N}^{(\mathrm{s})}(m \mid n)},
$$

where $v_{N}^{(\mathrm{s})}(m \mid n)$ is the cardinal of the set

$$
\Delta_{N}^{(\mathrm{s})}(m \mid n)=\left\{\boldsymbol{\delta}+\boldsymbol{\delta}^{\mathrm{R}} \mid \boldsymbol{\delta} \in \Delta_{N}(m \mid n)\right\} .
$$

Given a motif $\delta \in \Delta_{N}(m \mid n)$, we define its associated half-motif as the vector

$$
\hat{\boldsymbol{\delta}}= \begin{cases}\left(\delta_{1}+\delta_{N-1}, \delta_{2}+\delta_{N-2}, \ldots, \delta_{(N-1) / 2}+\delta_{(N+1) / 2}\right), & N \text { odd } \\ \left(\delta_{1}+\delta_{N-1}, \delta_{2}+\delta_{N-2}, \ldots, \delta_{(N-2) / 2}+\delta_{(N+2) / 2}, \delta_{N / 2}\right), & N \text { even } .\end{cases}
$$


Clearly, each vector $\delta+\delta^{\mathrm{R}}$ is uniquely determined by its corresponding half-motif $\hat{\delta}$, so that in order to evaluate $v_{N}^{(\mathrm{s})}(m \mid n)$ it suffices to count the number of distinct half-motifs. It is also important to observe that all the components of $\hat{\delta}$ can take the values $0,1,2$ except $\hat{\delta}_{N / 2}=\delta_{N / 2}$ (when $N$ is even), which can only take the values 0 or 1 .

In the genuinely supersymmetric case $\operatorname{su}(m \mid n)$ with $m n \neq 0$, it is straightforward to compute the number of distinct half-motifs, since in this case the motifs $\delta$ are arbitrary elements of $\{0,1\}^{N-1}$. Thus, when $N$ is odd the half-motif $\hat{\delta}$ is an arbitrary element of $\{0,1,2\}^{(N-1) / 2}$, and therefore

$$
v_{N}^{(\mathrm{s})}(m \mid n)=3^{(N-1) / 2} ; \quad m n \neq 0, \quad N \text { odd. }
$$

On the other hand, when $N$ is even we have

$$
v_{N}^{(\mathrm{s})}(m \mid n)=2 \cdot 3^{(N-2) / 2} ; \quad m n \neq 0, \quad N \text { even, }
$$

since in this case the last component of $\hat{\delta}$ can only take the values 0 or 1 . Hence the minimum average degeneracy of a translationally (and Yangian) invariant spin model in the genuinely supersymmetric case is given by

$$
d_{N, \min }^{(\mathrm{s})}= \begin{cases}\sqrt{3}\left(\frac{m+n}{\sqrt{3}}\right)^{N}, & N \text { odd } \\ \frac{3}{2}\left(\frac{m+n}{\sqrt{3}}\right)^{N}, & N \text { even. }\end{cases}
$$

From the previous discussion it is obvious that $d_{N, \text { min }}^{(\mathrm{s})}$ exactly coincides with the average degeneracy $d_{N}$ when there is no accidental degeneracy, namely when the function $f_{N}$ in Eq. (44) is injective.

Example 3. Consider the su( $m \mid n)$ elliptic model (28) in the special case $m=n=1$, which is obviously invariant under integer translations. As shown in Ref. [36], any spin chain of the form

$$
H=\sum_{i<j} h(i-j)\left(1-S_{i j}^{(1 \mid 1)}\right),
$$

where $h$ is an even $N$-periodic function, is isospectral to an su(1|1) Yangian-invariant spin model with linear energy function and dispersion relation

$$
\varepsilon_{N}(j)=\sum_{l=1}^{N-1}(1-\cos (2 \pi j l / N)) h(l) .
$$

Since $\varepsilon_{N}(j)=\varepsilon(N-j)$, the dispersion relation (51) is also translationally invariant in the sense of the definition (44). In particular, for the su(1|1) elliptic model (28) the dispersion relation can be computed in closed form using the techniques in Ref. [36], with the result

$$
\varepsilon_{N}(j)=\frac{N}{2}\left(1-\frac{E}{K}\right)+\frac{N^{2}}{4 K^{2}} \hat{\eta}_{1}-\frac{1}{16 K^{\prime 2}}\left[\wp(j / N)-\left(\zeta(j / N)-2 \eta_{1} \frac{j}{N}\right)^{2}\right], \quad j=1, \ldots, N-1
$$

(and $\varepsilon_{N}(0)=0$ ). In the previous formula $K^{\prime}=K\left(\sqrt{1-k^{2}}\right)$,

$$
E(k)=\int_{0}^{\pi / 2} \sqrt{1-k^{2} \sin ^{2} \varphi} \mathrm{d} \varphi
$$


is the complete elliptic integral of the second kind with modulus $k, \wp$ and $\zeta$ denote the Weierstrass elliptic functions with period lattice generated by 1 and $\mathrm{i} K /\left(N K^{\prime}\right), \eta_{1}=\zeta(1 / 2)$ and $\hat{\eta}_{1}=\zeta\left(1 / 2 ; 1 / 2, \mathrm{i} N K^{\prime} /(2 K)\right)$. Using Eqs. (10) and (52) we have computed the spectrum of the su(1|1) elliptic chain (28) for $N \leqslant 25$ and several values of the modulus $k$. In all cases, the average degeneracy $d_{N}$ coincides with the minimum average degeneracy (49) of a translationally (and Yangian) invariant spin model (cf. Fig. 47. Thus the spectrum of the su(1|1) elliptic chain does not exhibit any accidental degeneracy.

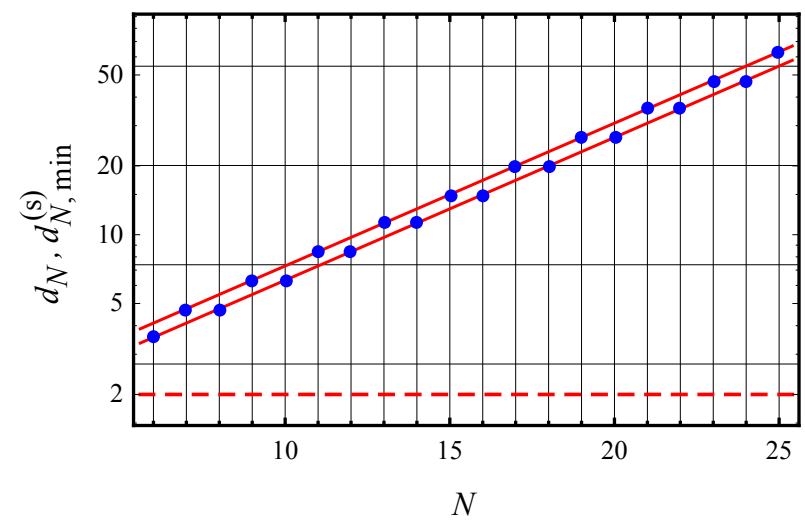

Figure 4: logarithmic plot of the average density of the $\mathrm{su}(1 \mid 1)$ elliptic chain with $k^{2}=1 / 2$ (blue dots), compared to the minimum average degeneracy [49] of a translationally (and Yangian) invariant su(1|1) spin model (continuous red lines, the top one for odd $N$ and the bottom one for even $N$ ). The dashed horizontal line represents the minimum average degeneracy [17] of a generic Yangian-invariant su(1|1) model.

In the non-supersymmetric (purely bosonic or fermionic) case, the computation of $d_{N, \text { min }}^{(\mathrm{s})}$ is considerably more involved, due to the fact that not all elements of $\{0,1\}^{N-1}$ are valid motifs. For this reason, we shall restrict ourselves in the rest of this section to the simplest and most important $\mathrm{su}(2)$ case. In the bosonic case (which, as we know, is equivalent to the fermionic one), an su(2) motif $\delta \in\{0,1\}^{N-1}$ cannot contain two consecutive 1's. Hence, for odd $N$ the corresponding half-motif $\hat{\boldsymbol{\delta}} \in\{0,1,2\}^{(N-1) / 2}$ satisfies the following two constraints:

i) No 2 can be preceded or followed by a 1 or a 2 .

ii) The last component of $\hat{\delta}$ cannot be equal to 2 .

It is also easy to see that any element of $\{0,1,2\}^{(N-1) / 2}$ satisfying these conditions is a valid halfmotif $\hat{\boldsymbol{\delta}}$ when $N$ is odd. On the other hand, when $N$ is even the last component of a half-motif is the middle component of the corresponding motif, and therefore condition ii) above should be replaced by the more stringent one

ii') The half-motif $\hat{\delta}$ can only end in 0 or 01 .

As before, it can be shown that any element of $\{0,1,2\}^{N / 2}$ satisfying conditions i) and ii') is a valid half-motif. Thus, in order to compute the minimum average degeneracy for odd (respectively even) $N$ we need only count the number of elements of $\{0,1,2\}^{(N-1) / 2}$ (respectively $\{0,1,2\}^{N / 2}$ ) satisfying conditions i) and ii) (respectively i) and $\left.\mathrm{ii}^{\prime}\right)$ ). 
Consider, to begin with, the case of odd $N=2 r+1$, and denote by $\mu_{r}$ the number of vectors $\hat{\delta} \in\{0,1,2\}^{r}$ satisfying the constraints i) and ii). Clearly, any such vector $\hat{\delta}$ can only end in $x 1$, $x 0$ and 20, where $x$ is either 0 or 1 . In the first two cases, the first $r-1$ components of $\hat{\delta}$ still satisfy conditions i) and ii), so that the number of valid half-motifs of length $r$ ending in $x 1$ or $x 0$ is equal to $2 \mu_{r-1}$. On the other hand, if $\hat{\boldsymbol{\delta}}$ ends in 20 its first $r-2$ components are a vector belonging to the set of half-motifs of length $r-2$ not ending in 1, whose cardinal is obviously $\mu_{r-2}-\mu_{r-3}$. We thus arrive at the recursion relation

$$
\mu_{r}=2 \mu_{r-1}+\mu_{r-2}-\mu_{r-3} .
$$

It is straightforward to check that $\mu_{1}=2, \mu_{2}=5$ and $\mu_{3}=11$, which, together with 53a, completely determines $\mu_{r}$ for all $r$. In fact, it is immediate to verify that the latter initial conditions are equivalent to the simpler ones

$$
\mu_{-2}=\mu_{-1}=0, \quad \mu_{0}=1 .
$$

Consider next the case of even $N=2 r$, and let $\tilde{\mu}_{r}$ denote the number of vectors $\hat{\delta} \in\{0,1,2\}^{r}$ satisfying the constraints $\mathrm{i}$ ) and $\left.\mathrm{ii}^{\prime}\right)$. The reader should easily convince himself that the valid motifs are precisely those from the $N=2 r+1$ case not ending in 11 , so that

$$
\tilde{\mu}_{r}=\mu_{r}-\mu_{r-2}
$$

Since $\mu_{r}$ (and hence $\mu_{r-2}$ ) is a solution of the linear recursion relation with constant coefficients 53a, it follows that $\tilde{\mu}_{r}$ satisfies the same recursion relation:

$$
\tilde{\mu}_{r}=2 \tilde{\mu}_{r-1}+\tilde{\mu}_{r-2}-\tilde{\mu}_{r-3} .
$$

However, the initial conditions are now $\tilde{\mu}_{1}=2, \tilde{\mu}_{2}=4, \tilde{\mu}_{3}=9$, or equivalently

$$
\tilde{\mu}_{-2}=\tilde{\mu}_{-1}=\tilde{\mu}_{0}=1 \text {. }
$$

Thus, the minimum average degeneracy of an su(2) translationally (and Yangian) invariant spin model is given by

$$
d_{N, \min }^{(\mathrm{s})}=\frac{2^{N}}{v_{N}^{(\mathrm{s})}(2)},
$$

where

$$
v_{N}^{(\mathrm{s})}(2)= \begin{cases}\mu_{(N-1) / 2}, & N \text { odd } \\ \tilde{\mu}_{N / 2}, & N \text { even. }\end{cases}
$$

The characteristic polynomial $p(\lambda)=\lambda^{3}-2 \lambda^{2}-\lambda+1$ of the recursion relation 53a- $55 \mathrm{a}$ has three real roots given by

$$
\lambda_{k}=\frac{2}{3}(1+\sqrt{7} \cos (\varphi+2 k \pi / 3)), \quad k=0,1,2,
$$

with

$$
\varphi \equiv \frac{1}{3} \arctan (3 \sqrt{3})
$$


As explained in Section 3.2, the sequences $\mu_{r}$ and $\tilde{\mu}_{r}(r=1,2, \ldots)$ can be expressed as

$$
\mu_{r}=\sum_{k=0}^{2} a_{k} \lambda_{k}^{r}, \quad \tilde{\mu}_{r}=\sum_{k=0}^{2} \tilde{a}_{k} \lambda_{k}^{r}
$$

where the coefficients $a_{k}$ and $\tilde{a}_{k}$ are respectively determined by the initial conditions (53b) and (55b). (In fact, from Eq. (54) it follows that $\tilde{a}_{k}=a_{k}\left(1-\lambda_{k}^{-2}\right)$.) Since the root of $p(\lambda)$ with largest absolute value is $\lambda_{0} \simeq 2.24698$ (while $\left|\lambda_{1,2}\right|<1$ ), the asymptotic behavior of $\mu_{r}$ and $\tilde{\mu}_{r}$ as $r \rightarrow \infty$ is given by

$$
\mu_{r} \underset{r \rightarrow \infty}{\simeq} a_{0} \lambda_{0}^{r}, \quad \tilde{\mu}_{r} \underset{r \rightarrow \infty}{\simeq} \tilde{a}_{0} \lambda_{0}^{r} .
$$

The coefficients $a_{0}$ and $\tilde{a}_{0}$ can be readily computed in closed form, with the result

$$
a_{0}=\frac{4(1+\sqrt{7} \cos \varphi)^{2}}{21(1+2 \cos 2 \varphi)} \simeq 0.97869, \quad \tilde{a}_{0}=\frac{4(1+\sqrt{7} \cos \varphi)^{2}-9}{21(1+2 \cos 2 \varphi)} \simeq 0.78485
$$

From Eqs. 57) and (58) we easily deduce the asymptotic behavior of the minimum average degeneracy $d_{N, \min }^{(\mathrm{s})}$ as $N \rightarrow \infty$ in the su(2) case, namely

$$
d_{N, \min }^{(\mathrm{s})} \underset{N \rightarrow \infty}{\simeq} \gamma_{2, N}^{(\mathrm{s})}\left(\frac{2}{\sqrt{\lambda}_{0}}\right)^{N}
$$

where the coefficient $\gamma_{2, N}^{(\mathrm{s})}$ is given by

$$
\gamma_{2, N}^{(\mathrm{s})}= \begin{cases}\frac{\sqrt{\lambda_{0}}}{a_{0}}, & N \text { odd } \\ \frac{1}{\tilde{a}_{0}}, & N \text { even }\end{cases}
$$

As $N \rightarrow \infty$, this minimum average degeneracy becomes considerably larger than the corresponding one for a generic Yangian-invariant su(2) model. Indeed, from Eqs. (42) and (60) we obtain

$$
\frac{d_{N, \min }^{(\mathrm{s})}}{d_{N, \text { min }}} \underset{N \rightarrow \infty}{\simeq} \alpha_{N}\left[\frac{2}{(\sqrt{5}-1) \sqrt{\lambda_{0}}}\right]^{N} \simeq \alpha_{N} \times 1.07941^{N},
$$

where $\alpha_{N}=2 \gamma_{2, N}^{(\mathrm{s})} /(5-\sqrt{5})$ is close to $1(1.10830$ for odd $N$ and 0.92197 for even $N)$.

\section{Average degeneracy of spin chains of Haldane-Shastry type}

Ever since Haldane's original paper [6], it has been noted that the spectrum of the HS chain exhibits a high degree of degeneracy, which is generally attributed to the Yangian symmetry of this model. In fact, since both the $\operatorname{su}(m \mid n)$ HS and PF chains are Yangian-invariant spin models, their average degeneracy is bounded below by $d_{N \text {,min }}$ in Eqs. (17) (in the genuinely supersymmetric case) or 27] (in the purely bosonic or fermionic case). In the case of the FI chain, although its symmetry under the Yangian has not been proved rigorously, since the spectrum is given by Eqs. (5) and (7) the same bounds apply. Roughly speaking, for the simplest su(2) case it can be checked that the average degeneracy of the HS, PF and FI chains is of the same order of 
magnitude as the above bounds - or, in the case of the HS chain, their refinement 56)- (57) for translationally invariant spin models- only for low values of $N$ (say, $N \lesssim 10$ ). In this section we shall study the average degeneracy of these models for much higher values of $N$ (up to $N=50$ ), and show that, contrary to widespread belief, it cannot be solely explained by their Yangian invariance.

As explained above, for simplicity's sake we shall restrict ourselves to the su(2) (bosonic or fermionic) case. For the PF chain, the computation of the average degeneracy is completely straightforward, since its spectrum is a set of consecutive integers [39] and the minimum and maximum energies can be found without difficulty. Indeed, consider (for instance) the bosonic case. The minimum energy is clearly zero, corresponding to the $\operatorname{su}(2)$ motif $\delta=(0, \ldots, 0)$. On the other hand, Eqs. (6) and (10) imply that the maximum energy is obtained from the motif $(\ldots, 0,1,0,1)$, with the result $E_{N, \max }=\left(N^{2}-\pi(N)\right) / 4$. Thus the number of distinct energy levels is given by

$$
v_{N}(2)=E_{N, \max }+1=\frac{N^{2}-\pi(N)}{4}+1 \quad(\mathrm{PF} \text { chain })
$$

and the average degeneracy is of course $d_{N}=2^{N} / v_{N}(2)$. In the case of the HS or FI chains, no simple way of expressing in closed form the number of distinct levels as a function of $N$ is known. For both of these chains, the most efficient way of computing the spectrum for relatively high values of $N$ is to evaluate the partition function [19,41] in a recursive way. More precisely, it can be shown that the partition function $Z_{N}$ of the (fermionic) FI chain is determined by the recursion relation

$$
Z_{N+1}(q)=2 q^{N(\alpha+N-1)} Z_{N}(q)+\left(1-q^{N(\alpha+N-1)}\right) q^{(N-1)(\alpha+N-2)} Z_{N-1}(q), \quad q \equiv \mathrm{e}^{-1 /\left(k_{\mathrm{B}} T\right)},
$$

with the initial conditions $Z_{-1}(q)=0, Z_{0}(q)=1$. The situation is a bit more involved for the HS chain, since its dispersion relation (6) depends explicitly on $N$. In this case, the (fermionic) partition function is given by $Z_{N}(q)=Q_{N}(q ; N)$, where $Q_{k}(q ; N)$ is obtained from the recursion relation

$$
Q_{k+1}(q ; N)=2 q^{k(N-k)} Q_{k}(q ; N)+\left(1-q^{k(N-k)}\right) q^{(k-1)(N-k+1)} Q_{k-1}(q ; N),
$$

with the initial conditions $Q_{-1}(q ; N)=0, Q_{0}(q ; N)=1$. Proceeding in this way it is feasible to compute the partition functions of the su(2) FI and HS chains for $N \leqslant 50$ in a standard desktop computer, and thus determine the number of distinct energies and the corresponding average degeneracy $d_{N}$. In Figure 5 we present logarithmic plots of $d_{N}$ for all three chains of Haldane-Shastry type with $N$ ranging from 10 to 50, compared to its lower bounds (27) (for the PF and FI chains) and (56)-(57) (for the HS chain). It is apparent from this figure that as $N$ becomes moderately large (of the order of 25) $d_{N}$ grows much faster than expected for a generic Yangian-invariant spin model. The ultimate reason for this behavior is the fact that these chains have a linear energy function whose dispersion relation is a low degree polynomial. Indeed, we shall show that this implies that the number of distinct levels has at most polynomial growth with $N$, compared to the typical exponential growth for a generic Yangian-invariant spin model; cf. Eqs. (16), 26)-(34), (47)-(48), (57)-(58). As a matter of fact, we have already proved that the number of distinct levels of the $\mathrm{su}(2) \mathrm{PF}$ chain is a second degree polynomial in $N$; see Eq. 62. We shall next prove an analogous result for the su(2) HS and FI chains.

In the case of the HS chain, since the dispersion relation $\varepsilon_{N}(j)$ is a polynomial in $j$ with integer coefficients, and $\varepsilon_{N}(j)>0$ for $1 \leqslant j \leqslant N-1$, the energies are clearly nonnegative integers. Moreover, from Eq. (6) it follows that all the energies are even when $N$ is odd. Since 

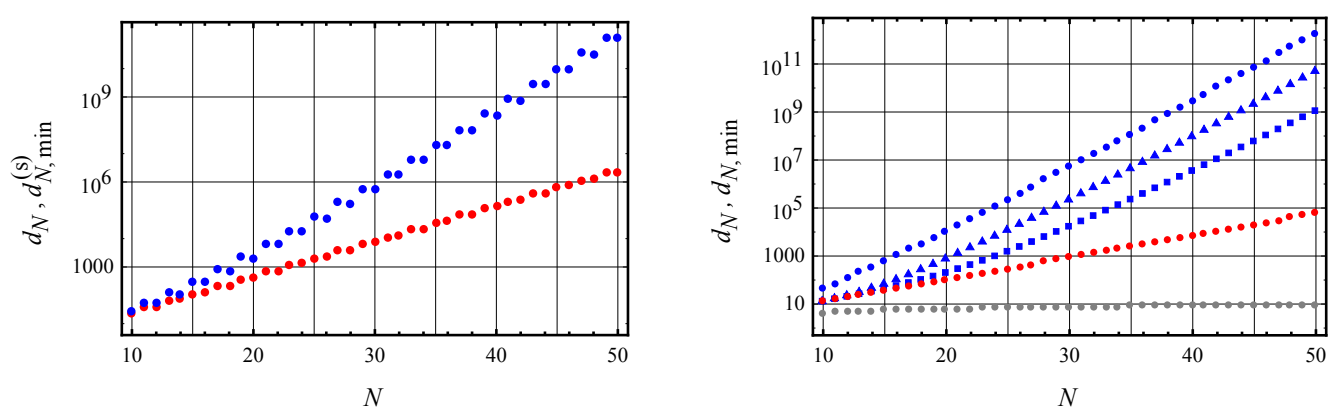

Figure 5: Left: logarithmic plot of the average degeneracy of the Haldane-Shastry su(2) spin chain (blue circles), compared to the minimum average degeneracy of a translationally (and Yangian) invariant spin model (red circles). Right: analogous plot for the su(2) Polychronakos-Frahm (blue circles) and Frahm-Inozemtsev (blue triangles for $\alpha=3$, blue squares for $\alpha$ irrational) spin chains, versus the minimum average degeneracy of a generic Yangian-invariant su(2) spin model (red circles) and of an su(2)-invariant spin chain of the form (3) (gray circles).

the minimum energy in the bosonic case is zero, the number of distinct energies satisfies the inequality

$$
v_{N}(2) \leqslant \frac{E_{N, \max }}{1+\pi(N)}+1,
$$

where $E_{N, \max }$ is the maximum energy of the bosonic chain. By Eqs. 22)-(23), this maximum energy is given by

$$
E_{N, \max }=\sum_{j=1}^{N-1} j(N-j)-E_{N, \min }^{(\mathrm{F})}=\frac{1}{6} N\left(N^{2}-1\right)-E_{N, \text { min }}^{(\mathrm{F})},
$$

where

$$
E_{N, \min }^{(\mathrm{F})}=\frac{1}{12} N\left(N^{2}-4+3 \pi(N)\right) .
$$

is the minimum energy of the fermionic chain computed in Ref. [21]. We thus obtain

$$
v_{N}(2) \leqslant\left\{\begin{array}{ll}
\frac{N}{12}\left(N^{2}+2\right)+1, & N \text { even } \\
\frac{N}{24}\left(N^{2}-1\right)+1, & N \text { odd }
\end{array} \quad \text { (HS chain) } .\right.
$$

In fact, as can be seen from Fig. 6, these bounds provide a good approximation to $v_{N}(2)$ for large $N$.

Finally, in the case of the FI chain, the behavior of $v_{N}(2)$ is quite different when $\alpha$ is rational or irrational. Indeed, if $\alpha=a / b$ is an irreducible rational number then $b E_{N}(\boldsymbol{\delta})$ is a nonnegative integer. Thus the number of distinct energies is bounded above by $b E_{N, \max }+1$, where $E_{N \text {, max }}$ is again the maximum energy of the bosonic chain. Proceeding as before and using the value of $E_{N, \text { min }}^{(\mathrm{F})}$ computed in Ref. [41] we readily obtain

$$
v_{N}(2) \leqslant\left\{\begin{array}{ll}
\frac{N}{12}\left(2 b N^{2}+3(a-b) N-2 b\right)+1, & N \text { even } \\
\frac{1}{12}\left(N^{2}-1\right)(2 b N+3(a-b))+1, & N \text { odd } \\
23 &
\end{array} \quad \text { (FI chain, } \alpha \in \mathbb{Q}\right) .
$$




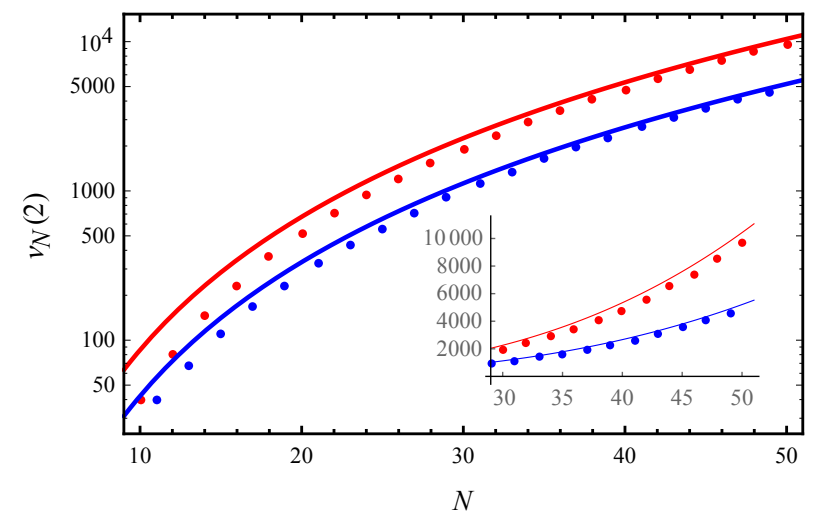

Figure 6: logarithmic plot of the number of distinct energy levels of the su(2) Haldane-Shastry chain (red circles for even $N$, blue circles for odd $N$ ), compared to the upper bounds 63 (solid lines). Inset: same plot for the range $30 \leqslant N \leqslant 50$, in ordinary (non-logarithmic) scale.

On the other hand, when $\alpha$ is irrational we can write

$$
E_{N}(\boldsymbol{\delta})=\alpha \sum_{j=1}^{N-1} j \delta_{j}+\sum_{j=1}^{N-1} j(j-1) \delta_{j} \equiv \alpha E_{N}^{(0)}(\boldsymbol{\delta})+E_{N}^{(1)}(\boldsymbol{\delta}),
$$

where the functions $E_{N}^{(i)}(\boldsymbol{\delta})(i=0,1)$ take nonnegative integer values and (in the bosonic case) actually vanish when $\delta$ is the zero motif. Hence

$$
v_{N}(2) \leqslant\left(E_{N, \max }^{(0)}+1\right)\left(E_{N, \text { max }}^{(1)}+1\right),
$$

where $E_{N, \max }^{(i)}$ is the maximum value of $E_{N}^{(i)}(\boldsymbol{\delta})$ over all valid su(2) bosonic motifs $\delta$. Although $E_{N, \max }^{(i)}$ can be computed without difficulty, for our purposes it suffices to note that

$$
E_{N, \max }^{(0)} \leqslant \sum_{j=1}^{N-1} j=\frac{N}{2}(N-1), \quad E_{N, \max }^{(0)} \leqslant \sum_{j=1}^{N-1} j(j-1)=\frac{N}{3}(N-1)(N-2),
$$

from which it easily follows that

$$
v_{N}(2) \leqslant \frac{N^{5}}{6} \quad(\text { FI chain, } \alpha \notin \mathbb{Q}) .
$$

Although the previous discussion has been restricted to the su(2) Haldane-Shastry type chains, its main conclusions extend to the whole class of $\operatorname{su}(m \mid n)$ Yangian-invariant spin models studied in Refs. [13, 33], whose energy function is of the form (10) with a dispersion relation $\varepsilon_{N}(j)$ polynomial in $j$ and $N$. Indeed, suppose that

$$
\varepsilon_{N}(j)=\sum_{(r, s) \in I} \alpha_{r s} N^{r} j^{s},
$$

where $I$ is a finite subset of $\mathbb{N}_{0} \times \mathbb{N}_{0}, \mathbb{N}_{0}$ being the set of nonnegative integers, and $\alpha_{r s}$ is independent of $N$ and non-zero for all $(r, s) \in I$. The energy function is then given by

$$
E_{N}(\boldsymbol{\delta})=\sum_{(r, s) \in I} \alpha_{r s} N^{r} \sum_{j=1}^{N-1} j^{s} \delta_{j} \equiv \sum_{(r, s) \in I} \alpha_{r s} N^{r} E_{N}^{(s)}(\boldsymbol{\delta}),
$$


where each function $E_{N}^{(s)}(\delta)$ takes nonnegative integer values and is bounded above by the power sum

$$
S_{N}^{(s)} \equiv \sum_{j=1}^{N-1} j^{s}=\frac{N^{s+1}}{s+1}+\mathrm{O}\left(N^{s}\right)
$$

We thus obtain the polynomial bound

$$
v_{N}(m \mid n) \leqslant \prod_{(r, s) \in I}\left(S_{N}^{(s)}+1\right)=\prod_{s}\left(S_{N}^{(s)}+1\right)^{r(s)},
$$

where $r(s)$ is the number of indices $r$ such that $(r, s) \in I$, and the last product ranges over all $s$ such that $r(s)>0$. Note that, on account of Eq. (67), the bound (68) satisfies

$$
\prod_{s}\left(S_{N}^{(s)}+1\right)^{r(s)}=\mathrm{O}\left(N^{\sum_{s}(s+1) r(s)}\right) .
$$

The bound in Eq. (68) can be considerably strengthened when all the coefficients $\alpha_{r s}$ in the dispersion relation 68 are rational numbers, as is the case with the PF and HS chains (or the FI chain, when $\alpha \in \mathbb{Q}$ ). Indeed, assume that $\alpha_{r s}=a_{r s} / b$, where $a_{r s} \in \mathbb{Z}$ and $b \in \mathbb{N}$. In this case $b E_{N}(\boldsymbol{\delta})$ takes only integer values, so that

$$
v_{N}(m \mid n) \leqslant b\left(E_{N, \max }-E_{N, \min }\right)+1,
$$

where $E_{N, \text { min }}$ and $E_{N, \text { max }}$ denote respectively the minimum and maximum energies. From the inequality

$$
\left|\varepsilon_{N}(j)\right| \leqslant \sum_{(r, s) \in I}\left|\alpha_{r s}\right| N^{r} j^{s} \leqslant N^{k} \sum_{(r, s) \in I}\left|\alpha_{r s}\right| \equiv A N^{k}, \quad 1 \leqslant j \leqslant N-1,
$$

where $k=\max _{(r, s) \in I}(r+s)$ is the total degree of $\varepsilon_{N}(j)$ as a polynomial in $(j, N)$, we immediately obtain

$$
E_{N, \max }-E_{N, \min } \leqslant 2 \max _{\delta \in \Delta_{N}(m \mid n)}\left|E_{N}(\boldsymbol{\delta})\right| \leqslant 2 N \max _{1 \leqslant j \leqslant N-1}\left|\varepsilon_{N}(j)\right| \leqslant 2 A N^{k+1},
$$

and hence

$$
v_{N}(m \mid n) \leqslant(2 b A+1) N^{k+1} .
$$

Note that this equation is in agreement with the asymptotic behavior of the bounds 62 - 64 for the PF, HS and FI chains (with $\alpha \in \mathbb{Q}$ in the latter case).

\section{Anyons and Fibonacci numbers}

The elementary excitations of certain quantum many-body systems can be described in terms of anyons, i.e., effective quasi-particles obeying neither the Bose-Einstein nor the Fermi-Dirac statistics [45-47]. The original definition of anyons is intrinsically two-dimensional, since it requires that the wave function change by a suitable phase under exchange of two identical particles, which is only consistent in two dimensions. An alternative definition of anyons in terms of a generalized exclusion principle was proposed by Haldane [14]. According to this definition, in a system of (identical, non-interacting) anyons the number of available one-particle states (or orbitals) decreases by $g \Delta k$ when the number of particles increases by $\Delta k$. The constant 
$g$, which must be a rational number, is called Haldane's exclusion parameter. Clearly, bosons and fermions have exclusion parameter $g=0$ and $g=1$, respectively. The advantage of Haldane's definition is that, in contrast with the original one, it is also valid in one dimension. For instance, it is well known that when the coupling constant is a nonnegative integer $a$ the spectrum of the $N$-particle Calogero model [29] is that of a system of $N$ free anyons with exclusion parameter $g=a$ in a harmonic oscillator potential (see, e.g., the review paper [48]).

Another important example of one-dimensional anyons is furnished by the su(2) HaldaneShastry chain, whose "spinon" excitations (in the antiferromagnetic case) are known to be anyons with $g=1 / 2$ [14]. It should be clear that this property is in fact shared by all su(2) (fermionic or bosonic) Yangian-invariant spin models, regardless of their energy function. To see this, consider an su(2) (say, bosonic) motif $\boldsymbol{\delta}$ and its associated border strip $\mathbf{k}=\left(k_{1}, \ldots, k_{r}\right)$, where $k_{1}+\cdots+k_{r}=$ $N$ is the number of spins. In the su(2) case, it is a simple matter to compute the dimension of the corresponding $Y(\mathrm{gl}(2 \mid 0))$-module $V_{\mathbf{k}}(2 \mid 0) \equiv V_{\mathbf{k}}$. Indeed, a (bosonic) su(2) border strip $\mathbf{k}$ cannot contain rows with more than two boxes. Moreover, any tableau corresponding to $\mathbf{k}$ must necessarily contain the sequence 01 (from left to right) in any row with two boxes. The dimension of $V_{\mathbf{k}}$ is thus given by the number of ways of filling the remaining ("empty") boxes in each column of the border strip $\mathbf{k}$ (from top to bottom) with sequences of the form $0 \cdots 01 \cdots 1$ (where the number of 0 's or 1's can of course be zero). Thus the $r$ columns of $\mathbf{k}$ play the role of available orbitals, and its $N-2 r+2$ empty boxes that of particles with two internal degrees of freedom ( 0 or 1$)$. (These particles are obviously bosons, since each orbital can be occupied by more than one particle with the same quantum numbers.) Thus in this case we have $k=N-2 r+2$ and $\Delta r=-\Delta k / 2$, so that Haldane's definition is satisfied with $g=1 / 2$. This shows that any su(2) Yangian-invariant model is indeed equivalent to a system of anyons with two internal degrees of freedom and exclusion parameter $g=1 / 2$ (semions, in Haldane's terminology).

Haldane's definition of anyons can be equivalently formulated in terms of the total number of states in a system with $k$ anyons and $\mathcal{N}$ one-particle states (or orbitals). Indeed, for anyons with exclusion parameter $g$ this number is given by

$$
w_{k}(g)=\left(\begin{array}{c}
\mathcal{N}-(g-1)(k-1) \\
k
\end{array}\right) .
$$

When $g$ is a natural number, a very simple realization of this statistics can be obtained by considering a system of $k$ particles and $\mathcal{N}$ orbitals satisfying the following generalized Pauli exclusion principle: two occupied orbitals must be separated by at least $g-1$ empty ones. This requirement is clearly reminiscent of the rule satisfied by the bosonic su(2) motifs. More precisely, we can interpret such a motif $\delta \in \Delta_{N}(2 \mid 0)$ as encoding a state of a quantum system with $N-1$ orbitals (the components of $\boldsymbol{\delta}$ ), with a 1 indicating an occupied orbital and 0 an empty one. With this interpretation, the 1's in the motif $\delta$ are quasi-particles satisfying the generalized Pauli principle with $g=2$, and thus can be regarded as anyons with exclusion parameter $g=2$. This point of view is particularly natural when the energy function is linear (cf. Eq. (10)), as is the case with spin chains of Haldane-Shastry type (cf. Eqs. (5)-(7)). Indeed, for such Yangian-invariant models the energy function can be written as

$$
E_{N}(\boldsymbol{\delta})=\sum_{i=1}^{r-1} \varepsilon_{N}\left(K_{i}\right),
$$

where $K_{i}$ is the position of the $i$-th 1 in $\delta$. It should be stressed that this interpretation is not equivalent to the one discussed above in terms of spinons, for which $g=1 / 2$. Indeed, in the 
latter interpretation the 1's in the motif (or, more accurately, the sequences $(0,1)$ ) simply separate the orbitals, while the remaining 0's correspond to the particles. In particular, a fundamental difference between both interpretations is that in the new one the degeneracy associated to each motif (that is, the dimension of the corresponding invariant module $V_{\mathbf{k}}$ ) is not taken into account. For this reason, the new interpretation defines a different type of system, whose Hilbert space dimension equals the number $v_{N}(2)$ of distinct $\operatorname{su}(2)$ motifs. Since $v_{N}(2)$ is given by the Fibonacci number $F_{N+1}$ (cf. Eq. (26)), from Eq. (70) with $\mathcal{N}=N-1$ and $g=2$ we obtain the remarkable identity

$$
F_{N+1}=\sum_{k=0}^{\lfloor N / 2\rfloor}\left(\begin{array}{c}
N-k \\
k
\end{array}\right),
$$

where the upper limit is determined by the condition $N-k \geqslant k$. In other words, the $(N+1)$-th Fibonacci number is obtained by diagonally summing the binomial coefficients arranged in a Pascal triangle [34]. This classical number-theoretic result can thus be interpreted as expressing the dimension of the Hilbert space of a system of $g=2$ anyons with $N-1$ orbitals in terms of the corresponding dimensions of its $k$-particle subspaces.

Motivated by the previous discussion, we shall next consider the bosonic $\operatorname{su}(m)$ motifs with $m>2$ as representing the states of a system of quasi-particles (namely, the 1's in the motif) with $N-1$ orbitals (the motif's components), subject to the exclusion principle that there can be no more than $m-1$ consecutive occupied orbitals. As before, this rule obviously does not take into account the degeneracy associated to each motif, so that the system under consideration is not an $\operatorname{su}(m)$ Yangian-invariant spin model. It can instead be regarded as an effective model, whose spectrum (71) reproduces the set of distinct energy levels of a generic (bosonic) $\mathrm{su}(m)$ Yangian-invariant spin model with linear energy function and dispersion relation $\varepsilon_{N}(j)$.

We shall next compute the statistical weight $w_{k}$ for the new system just introduced. To this end, let $\delta \in \Delta_{N}(m \mid 0)$ be a bosonic su $(m)$ motif with $j_{0}$ single 1's (i.e., $(1,0)$ sequences), $j_{1}$ double 1 's (i.e, $(1,1,0)$ sequences $), \ldots$, and $j_{m-2}$ sequences $(\underbrace{1, \ldots, 1}_{m-1}, 0)$, with

$$
j_{0}+2 j_{1}+\cdots+(m-1) j_{m-2}=k .
$$

Removing the zero in each sequence $(1,0),(1,0)$ in each sequence $(1,1,0), \ldots,(\underbrace{1, \ldots, 1}_{m-2}, 0)$ in each sequence $(\underbrace{1, \ldots, 1}_{m-1}, 0)$, except for the zero (if any) following the last 1 in the motif, we are left with a sequence $\bar{\delta} \in\{0,1\}^{N-k}$ containing $j_{0}+\cdots+j_{m-2} 1$ 's. Conversely, it is clear that from any such vector $\bar{\delta} \in\{0,1\}^{N-k}$ one can construct a bosonic su(m) motif with $j_{0}$ single 1 's, $j_{1}$ double 1 's, $\ldots$, and $j_{m-2}$ sequences $(\underbrace{1, \ldots, 1}_{m-1}, 0)$ by adding a zero after $j_{0} 1$ 's, the sequence $(1,0)$ after $j_{1}$ of the remaining 1 's, etc. Thus, the number $w\left(j_{0}, \ldots, j_{m-2}\right)$ of motifs with $j_{0}$ single 1 's, $j_{1}$ double 1's, etc., is given by the number of ways of choosing the positions of the $j_{0} 1$ 's starting a sequence $(1,0)$, the $j_{1} 1$ 's starting a sequence $(1,1,0)$, etc., among the integers $1, \ldots, N-k$. In other words,

$$
w\left(j_{0}, \ldots, j_{m-2}\right)=\frac{(N-k) !}{\left(N-k-\sum_{i=0}^{m-2} j_{i}\right) ! \prod_{i=0}^{m-2} j_{i} !} .
$$


The statistical weight $w_{k}$ is the sum of all $w\left(j_{0}, \ldots, j_{m-2}\right)$ whose arguments $\left(j_{0}, \ldots, j_{m-2}\right)$ satisfy (73), namely

$$
w_{k}=\sum_{\substack{j_{0}, \ldots, j_{m-2} \geqslant 0 \\ j_{0}+2 j_{1}+\cdots+(m-1) j_{m-2}=k}} \frac{(N-k) !}{\left(N-k-\sum_{i=0}^{m-2} j_{i}\right) ! \prod_{i=0}^{m-2} j_{i} !} .
$$

Note that for $m=2$ this equation obviously reduces to Eq. (70) with $g=2$ and $\mathcal{N}=N-1$. Eliminating $j_{0}$ with the help of Eq. (73) and explicitly enforcing the condition $\sum_{i=0}^{m-2} j_{i} \leqslant N-k$ arising from the first factor in the denominator we obtain the equivalent expression

$$
w_{k}=\sum_{\substack{j_{1}, \ldots, j_{m-2} \geq 0 \\ 2 j_{1}+3 j_{2}+\cdots+(m-1) j_{m-2} \leqslant k \\ j_{1}+2 j_{2}+\cdots+(m-2) j_{m-2} \geqslant 2 k-N}} \frac{(N-k) !}{\left(N-2 k+\sum_{i=1}^{m-2} i j_{i}\right) !\left(k-\sum_{i=1}^{m-2}(i+1) j_{i}\right) ! \prod_{i=1}^{m-2} j_{i} !} .
$$

For instance, for $m=3$ we have

$$
w_{k}=\sum_{j=\max (2 k-N, 0)}^{\lfloor k / 2\rfloor} \frac{(N-k) !}{(N-2 k+j) !(k-2 j) ! j !}, \quad m=3 .
$$

The total dimension of the system's Hilbert space, which is the same as the number $v_{N}(m)$ of bosonic $\operatorname{su}(m)$ motifs for a Yangian-invariant spin model with $N$ spins, is obtained by summing the statistical weight $w_{k}$ over all possible values of $k$. If $N-1=r m+s$ with $0 \leqslant s \leqslant m-1$, the maximum value of $k$ is clearly

$$
r(m-1)+s=\frac{m-1}{m}(N-1-s)+s=\frac{m-1}{m} N-\frac{m-1-s}{m}=\left\lfloor\frac{m-1}{m} N\right\rfloor,
$$

since $0 \leqslant m-1-s \leqslant m-1$. From Eqs. (75) and (26) we finally obtain the identity

$$
F_{N+m-1}^{(m)}=\sum_{k=0}^{\lfloor(m-1) N / m\rfloor} \sum_{\substack{j_{1}, \ldots, j_{m-2} \geqslant 0 \\ 2 j_{1}+3 j_{2}+\cdots+(m-1) j_{m-2} \leq k \\ j_{1}+2 j_{2}+\cdots+(m-2) j_{m-2} \geqslant 2 k-N}} \frac{(N-k) !}{\left(N-2 k+\sum_{i=1}^{m-2} i j_{i}\right) !\left(k-\sum_{i=1}^{m-2}(i+1) j_{i}\right) ! \prod_{i=1}^{m-2} j_{i} !} .
$$

An alternative version of the latter equation follows by using Eq. (74) for $w_{k}$ and eliminating $k$ using Eq. 73), namely

$$
F_{N+m-1}^{(m)}=\sum_{\substack{j_{0}, \ldots, j_{m-2} \geqslant 0 \\ 2 j_{0}+3 j_{1}+\cdots+\cdots+m j_{m-2} \leqslant N}} \frac{\left(N-\sum_{i=0}^{m-2}(i+1) j_{i}\right) !}{\left(N-\sum_{i=0}^{m-2}(i+2) j_{i}\right) ! \prod_{i=0}^{m-2} j_{i} !} .
$$

This is essentially the classical combinatorial expression for the generalized Fibonacci numbers proved in [34] (cf. Eq. (28") in the latter reference).

It is apparent from Eqs. (70) (with $\mathcal{N}=N-1$ ) and (75) that for $m>2$ the quasi-particles associated to the (bosonic) $\mathrm{su}(\mathrm{m})$ motifs of a Yangian-invariant spin model are not anyons. Note, however, that there is a generalization of Haldane's definition (70), due to Murthy and Shankar [49], to systems with an infinite-dimensional one-particle Hilbert space. The latter generalization is 
essentially based on the behavior of the statistical weight 70 as the number of orbitals $\mathcal{N}$ tends to infinity for any fixed $k$, namely

$$
w_{k}(g)=\frac{\mathcal{N}^{k}}{k !}+\left(\frac{1}{2}-g\right) \frac{\mathcal{N}^{k-1}}{(k-2) !}+\mathrm{O}\left(\mathcal{N}^{k-2}\right),
$$

from which it follows that

$$
\frac{1}{2}-g=\lim _{\mathcal{N} \rightarrow \infty} \frac{\mathcal{N}}{k(k-1)}\left(\frac{k ! w_{k}(g)}{\mathcal{N}^{k}}-1\right)
$$

This observation suggests looking at the asymptotic behavior of the density of states 75 as $\mathcal{N}=N-1 \rightarrow \infty$ with $k$ fixed. To this end, note first of all that $j_{i} \leqslant k /(i+1)$ on account of the restriction $\sum_{i=1}^{m-2}(i+1) j_{i} \leqslant k$, and that

$$
\frac{(\mathcal{N}-k+1) !}{(\mathcal{N}-2 k+l+1) !} \underset{\mathcal{N} \rightarrow \infty}{=} \mathcal{N}^{k-l}+\mathrm{O}\left(\mathcal{N}^{k-l-1}\right) .
$$

Thus the leading terms in the sum $(75)$ are obtained for $j_{1}=\cdots=j_{m-2}=0$ and $j_{1}=1$, $j_{2}=\cdots=j_{m-2}=0$, or more precisely

$w_{k}=\frac{(\mathcal{N}-k+1) !}{(\mathcal{N}-2 k+1) ! k !}+\frac{(\mathcal{N}-k+1) !}{(\mathcal{N}-2 k+2) !(k-2) !}+\mathrm{O}\left(\mathcal{N}^{k-2}\right)=\left(\begin{array}{c}\mathcal{N}-k+1 \\ k\end{array}\right)+\frac{\mathcal{N}^{k-1}}{(k-2) !}+\mathrm{O}\left(\mathcal{N}^{k-2}\right)$.

Using Eqs (70)-(78) with $g=2$ to expand the first term on the RHS we immediately obtain

$$
w_{k}=\frac{\mathcal{N}^{k}}{k !}-\frac{1}{2} \frac{\mathcal{N}^{k-1}}{(k-2) !}+\mathrm{O}\left(\mathcal{N}^{k-2}\right) \text {. }
$$

Thus as $\mathcal{N} \rightarrow \infty$ with $k$ fixed the density of states (75) satisfies Eq. (78) with $g=1$. Note that, as already observed by Murthy and Shankar, if the system's maximum and minimum energies are independent of $\mathcal{N}$ (or, more generally, the spectrum is bounded by $\mathcal{N}$-independent constants) the limit $\mathcal{N} \rightarrow \infty$ with $k$ fixed can be naturally interpreted as the continuum limit. We conclude that in the continuum limit the (bosonic) su( $m$ ) motifs with $m>2$ behave as a system of fermions, according to Murthy and Shankar's definition.

\section{Conclusions}

In this paper we consider a wide class of finite-dimensional Yangian-invariant quantum models — which we have called Yangian-invariant spin models - closely related to representations of the Yangian $Y(\operatorname{gl}(m \mid n))$ labeled by border strips. This class includes several widely studied systems, such as spin chains of Haldane-Shastry type and certain families of one-dimensional vertex models. A common feature of all Yangian-invariant spin models is the relatively high degeneracy of their spectrum, essentially due to their symmetry under the Yangian. As a quantitative measure of the degree of degeneracy of these models, we compute in closed form the intrinsic average degeneracy of any such model stemming from its Yangian invariance. It turns out that, in the non-supersymmetric case, this minimum average degeneracy is expressed in terms of generalized Fibonacci numbers. Using several fundamental properties of these numbers, we derive the asymptotic behavior of the minimum average degeneracy as the number of spins tends to infinity. Our results provide a stringent test for a quantum system to be a Yangian-invariant 
spin model. As an example, we apply this test to the $\operatorname{su}(m \mid n)$ supersymmetric version of Inozemtsev chain, which has been studied in the context of the gauge-string duality as a candidate for reproducing the spectrum of the dilation operator at several loops; see, e.g., [50]. We have also refined the previous results on the minimum average degeneracy for models that are in addition translationally invariant, both in the $\operatorname{su}(m \mid n)$-supersymmetric and in the simplest su(2) cases.

The second main problem that we address in this paper is the analysis of the precise role played by the Yangian symmetry in the high degeneracy of the spectra of spin chains of HaldaneShastry type. We show that these chains are much more degenerate than generic Yangianinvariant spin models, since the number of distinct levels grows polynomially for the former systems and exponentially for the latter. This accidental degeneracy is essentially due to the polynomial character of the dispersion relation, and is therefore shared by the family of onedimensional vertex models studied in Refs. [13, 33]. Moreover, if the coefficients in the dispersion relation are rational numbers (as is the case for the HS and PF chains), we show that the accidental degeneracy is even higher, as illustrated by the FI chain with a rational vs. irrational parameter. Finally, we consider an effective model of quasi-particles describing the distinct energy levels of a non-supersymmetric Yangian-invariant spin model. We show that in the su(2) case these quasi-particles are anyons with exclusion parameter $g=2$, while in the $\operatorname{su}(m)$ case with $m>2$ they become fermions in the continuum limit. We also provide a simple interpretation of several combinatorial expressions for $m$-nacci numbers in terms of the statistical weights of the effective model of $\operatorname{su}(m)$ quasi-particles.

\section{Acknowledgments}

This work was supported in part by Spain's MINECO under grant no. FIS2011-22566 and by the Universidad Complutense and Banco Santander under grant no. GR3/14-910556.

\section{References}

[1] A. N. Kirillov, A. Kuniba, T. Nakanishi, Commun. Math. Phys. 185 (1997) 441-465.

[2] M. Nazarov, V. Tarasov, J. Reine Angew. Math 496 (1998) 181-212.

[3] F. D. M. Haldane, Z. N. C. Ha, J. C. Talstra, D. Bernard, V. Pasquier, Phys. Rev. Lett. 69 (1992) 2021-2025.

[4] K. Hikami, Nucl. Phys. B 441 (1995) 530-548.

[5] P. Bouwknegt, K. Schoutens, Nucl. Phys. B 482 (1996) 345-372.

[6] F. D. M. Haldane, Phys. Rev. Lett. 60 (1988) 635-638.

[7] B. S. Shastry, Phys. Rev. Lett. 60 (1988) 639-642.

[8] F. D. M. Haldane, in: A. Okiji, N. Kawakami (Eds.), Correlation Effects in Low-dimensional Electron Systems, volume 118 of Springer Series in Solid-state Sciences, pp. 3-20.

[9] A. P. Polychronakos, Phys. Rev. Lett. 70 (1993) 2329-2331.

[10] H. Frahm, J. Phys. A: Math. Gen. 26 (1993) L473-L479.

[11] K. Hikami, B. Basu-Mallick, Nucl. Phys. B 566 (2000) 511-528.

[12] D. Bernard, M. Gaudin, F. D. M. Haldane, V. Pasquier, J. Phys. A: Math. Gen. 26 (1993) 5219-5236.

[13] B. Basu-Mallick, N. Bondyopadhaya, K. Hikami, SIGMA 6 (2010) 091(13).

[14] F. D. M. Haldane, Phys. Rev. Lett. 67 (1991) 937-940.

[15] F. D. M. Haldane, Phys. Rev. Lett. 66 (1991) 1529-1532.

[16] B. Basu-Mallick, N. Bondyopadhaya, D. Sen, Nucl. Phys. B 795 (2008) 596-622.

[17] J. I. Cirac, G. Sierra, Phys. Rev. B 81 (2010) 104431(4).

[18] A. E. B. Nielsen, J. I. Cirac, G. Sierra, J. Stat. Mech.-Theory E. (2011) P11014(39).

[19] F. Finkel, A. González-López, Phys. Rev. B 72 (2005) 174411(6).

[20] B. Basu-Mallick, N. Bondyopadhaya, Nucl. Phys. B 757 (2006) 280-302.

[21] J. C. Barba, F. Finkel, A. González-López, M. A. Rodríguez, Europhys. Lett. 83 (2008) 27005(6)

[22] J. C. Barba, F. Finkel, A. González-López, M. A. Rodríguez, Nucl. Phys. B 806 (2009) 684-714. 
[23] D. Giuliano, A. Sindona, G. Falcone, F. Plastina, L. Amico, New J. Phys. 12 (2010) 025022(15).

[24] N. Kawakami, Phys. Rev. B 46 (1992) 1005-1014.

[25] Z. N. C. Ha, F. D. M. Haldane, Phys. Rev. B 47 (1993) 12459-12469.

[26] B. Sutherland, Phys. Rev. A 4 (1971) 2019-2021.

[27] B. Sutherland, J. Math. Phys. 12 (1971) 246-250.

[28] Z. N. C. Ha, F. D. M. Haldane, Phys. Rev. B 46 (1992) 9359-9368.

[29] F. Calogero, J. Math. Phys. 12 (1971) 419-436.

[30] J. A. Minahan, A. P. Polychronakos, Phys. Lett. B 302 (1993) 265-270.

[31] V. I. Inozemtsev, Phys. Scr. 53 (1996) 516-520.

[32] H. Frahm, V. I. Inozemtsev, J. Phys. A: Math. Gen. 27 (1994) L801-L807.

[33] P. Banerjee, B. Basu-Mallick, J. Math. Phys. 53 (2012) 083301.

[34] E. P. Miles, Jr, Am. Math. Mon. 67 (1960) 745-752.

[35] V. I. Inozemtsev, J. Stat. Phys. 59 (1990) 1143-1155.

[36] F. Finkel, A. González-López, J. Stat. Mech.-Theory E. (2014) P12014(28).

[37] M. L. Nazarov, Lett. Math. Phys. 21 (1991) 123-131.

[38] B. Basu-Mallick, N. Bondyopadhaya, K. Hikami, D. Sen, Nucl. Phys. B 782 (2007) 276-295.

[39] A. P. Polychronakos, Nucl. Phys. B 419 (1994) 553-566.

[40] B. Basu-Mallick, H. Ujino, M. Wadati, J. Phys. Soc. Jpn. 68 (1999) 3219-3226.

[41] J. C. Barba, F. Finkel, A. González-López, M. A. Rodríguez, Nucl. Phys. B 839 (2010) 499-525.

[42] F. Finkel, A. González-López, Ann. Phys.-New York 351 (2014) 797-827.

[43] W. R. Spickerman, R. N. Joyner, Fibonacci Quart. (1984) 327-331.

[44] G. P. B. Dresden, Z. Du, J. Integer Seq. 17 (2014) 14.4.7(9).

[45] J. M. Leinaas, J. Myrheim, Nuovo Cimento 37B (1977) 1-23.

[46] F. Wilczek, Phys. Rev. Lett. 48 (1982) 2-4.

[47] F. Wilczek, Phys. Rev. Lett. 49 (1982) 957-959.

[48] A. P. Polychronakos, J. Phys. A: Math. Gen. 39 (2006) 12793-12845.

[49] M. V. N. Murthy, R. Shankar, Phys. Rev. Lett. 72 (1994) 3629-3633.

[50] N. Beisert (Ed.), Special Volume: Review on AdS/CFT Integrability, volume 99 of Lett. Math. Phys., Springer, 2012 . 